\title{
Nickel-Catalyzed Cycloaddition of Unsaturated Hydrocarbons and Carbonyl Compounds
}

\author{
Thomas N. Tekavec and Janis Louie* \\ Department of Chemistry, University of Utah, 315 South 1400 East, Salt Lake City, UT 84112-0850
}

\section{SUPPORTING INFORMATION}

\section{General Experimental}

All reactions were conducted under an atmosphere of $\mathrm{N}_{2}$ using standard Schlenk techniques or in a $\mathrm{N}_{2}$ filled glove-box unless otherwise noted. Toluene was dried over neutral alumina under $\mathrm{N}_{2}$ using a Grubbs-type solvent purification system. THF was freshly distilled from Na/benzophenone. Ni(COD) $)_{2}$ was purchased from Strem and used without further purification. SIPr, IPr, IMes and ItBu ligands were prepared as previously reported. ${ }^{1,2,3}$ Diynes 2,2-di-but-2-ynyl-malonic acid dimethyl ester (4a) and 2,2-di-prop-2-ynyl-malonic acid dimethyl ester (4b) were synthesized by literature procedures or under slightly modified conditions. ${ }^{4,5}$ Sodium hydride was thoroughly washed with pentane and dried in vacuo prior to use. All other reagents were purchased and used without further purification unless otherwise noted.

${ }^{1} \mathrm{H}$ and ${ }^{13} \mathrm{C}$ Nuclear Magnetic Resonance spectra of pure compounds were acquired at 300 and $75 \mathrm{MHz}$, respectively unless otherwise noted. All spectra are referenced to a singlet at $7.27 \mathrm{ppm}$ for ${ }^{1} \mathrm{H}$ and to the center line of a triplet at $77.23 \mathrm{ppm}$ for ${ }^{13} \mathrm{C}$. The abbreviations s, d, dd, dt, dq, t, td, tq q, qt, quint, sept, septd, septt, m, brm, brd, brt, and brs stand for singlet, doublet, doublet of doublets, doublet of triplets, doublet of quartets, triplet, triplet of doublets, triplet of quartets, quartet, quartet of triplets, quintet, septet, septet of doublets, septet of triplets, multiplet, broad multiplet, broad doublet, broad triplet, and broad singlet, in that order. All ${ }^{13} \mathrm{C}$ NMR spectra were proton decoupled. IR spectra were recorded on a Bruker Tensor 27 FT-IR spectrometer. HRMS were performed at the mass spectrometry facility at The University of Utah.
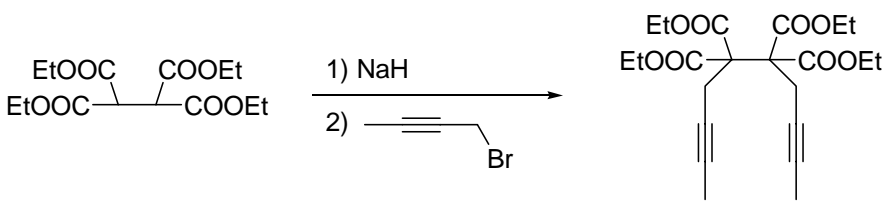
Preparation of 2-but-2-ynyl-2,3,3-tris-ethoxycarbonyl-hept-5-ynoic acid ethyl ester (1a). To a stirred suspension of $\mathrm{NaH}(255.0 \mathrm{mg}, 10.6 \mathrm{mmol})$ and dry THF (40 mL) in a $100 \mathrm{~mL}$ RBF was added tetraethyl 1,1,2,2ethanetetracarboxylate $(1.3000 \mathrm{~g}, 4.1 \mathrm{mmol})$ under $\mathrm{N}_{2}$ counter-flow in two portions. The resulting solution was stirred at room temperature for $1 \mathrm{~h}$ after which time 1-bromo-2-butyne (1.409 g, $10.6 \mathrm{mmol})$ was added. The reaction mixture was then heated at reflux for 44 hrs at which time GC analysis showed no remaining starting ester or mono-alkylated ester. The solution was cooled to room temperature and quenched with $20 \mathrm{~mL}$ sat. $\mathrm{NH}_{4} \mathrm{Cl}$ solution. The aqueous layer was extracted $3 \times 10 \mathrm{~mL} \mathrm{Et}_{2} \mathrm{O}$. The collected organics were washed with brine, dried with anhydrous $\mathrm{Na}_{2} \mathrm{SO}_{4}$, and concentrated in vacuo. The resulting crude yellow oil was purified by flash column chromatography (15\% EtOAc/hexanes then 25\% EtOAc/hexanes) to yield a pale yellow oil which was triturated with hexanes to afford 1a (1.4848g, 86\%) as a white solid. ${ }^{1} \mathrm{H}$ and ${ }^{13} \mathrm{C}$ NMR were identical to those reported in literature. ${ }^{6}$

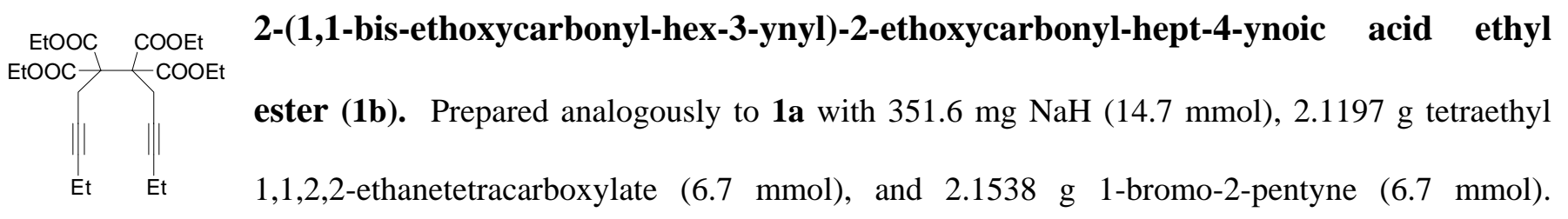
Purified by column chromatography eluting with 15\% EtOAc/hexanes to yield $\mathbf{1 b}(2.4211 \mathrm{~g}, 81 \%)$ as a yellow oil. ${ }^{1} \mathrm{H}$ NMR (300 MHz, $\mathrm{CDCl}_{3}$ ): $\delta(\mathrm{ppm})$ 4.30-4.16 (m, 8H), 3.10 (t, $\left.J=2.2 \mathrm{~Hz}, 4 \mathrm{H}\right), 2.12$ (qt, $J=7.5,2.2 \mathrm{~Hz}, 4 \mathrm{H}$ ), $1.29(\mathrm{t}, J=7.1 \mathrm{~Hz}, 12 \mathrm{H}), 1.08(\mathrm{t}, J=7.5 \mathrm{~Hz}, 6 \mathrm{H}) .{ }^{13} \mathrm{C}\left\{{ }^{1} \mathrm{H}\right\} \mathrm{NMR}\left(75 \mathrm{MHz}, \mathrm{CDCl}_{3}\right): \delta(\mathrm{ppm})$ 168.8, 84.0, 75.1, 62.0, 61.8, 22.8, 14.0, 13.9, 12.5. IR (neat): 2981, 1734, 1448, 1368, 1206, 1097, $1039 \mathrm{~cm}^{-1}$. HRMS(EI): calcd for $\mathrm{C}_{24} \mathrm{H}_{35} \mathrm{O}_{8}\left(\mathrm{MH}^{+}\right)$451.2332, obsd 451.2328.

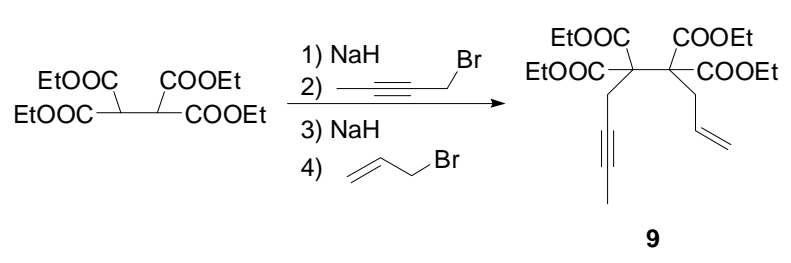

Preparation of 2-allyl-2,3,3-tris-ethoxycarbonyl-hept-5-ynoic acid ethyl ester (9). To a stirred suspension of $\mathrm{NaH}$ (356.0 mg, $14.9 \mathrm{mmol})$ and dry THF (135 mL) in a $250 \mathrm{~mL}$ RBF was added tetraethyl 1,1,2,2ethanetetracarboxylate (4.2970 g, $13.5 \mathrm{mmol}$ ) under $\mathrm{N}_{2}$ counter-flow in two portions. The resulting solution was stirred at room temperature for $3 \mathrm{hrs}$ after which time 1-bromo-2-butyne (1.9748 g, $14.9 \mathrm{mmol}$ ) was added. The 
reaction mixture was then stirred at room temperature for 3 hrs then heated at reflux for 8 hrs at which time GC analysis showed no remaining starting ester. The solution was cooled to room temperature and quenched with 20 mL sat. $\mathrm{NH}_{4} \mathrm{Cl}$ solution. The aqueous layer was extracted $3 \times 10 \mathrm{~mL} \mathrm{Et}_{2} \mathrm{O}$. The collected organics were washed with brine, dried with anhydrous $\mathrm{Na}_{2} \mathrm{SO}_{4}$, and concentrated in vacuo. The resulting crude yellow oil was purified by flash column chromatography (15\% EtOAc/hexanes then 25\% EtOAc/hexanes) to yield 2,3,3-trisethoxycarbonyl-hept-5-ynoic acid ethyl ester (4.5511 g, 91\%) as a pale yellow oil. ${ }^{1} \mathrm{H}$ NMR (300 MHz, $\left.\mathrm{CDCl}_{3}\right): \delta$ (ppm) 4.34 (s, 1H), 4.30-4.17 (m, 8H), 2.93 (q, $J=2.6 \mathrm{~Hz}, 2 \mathrm{H}), 1.78$ (t, $J=2.6 \mathrm{~Hz}, 3 \mathrm{H}), 1.28$ (t, $J=7.2 \mathrm{~Hz}, 12 \mathrm{H})$. ${ }^{13} \mathrm{C}\left\{{ }^{1} \mathrm{H}\right\}$ NMR (75 MHz, $\left.\mathrm{CDCl}_{3}\right): \delta(\mathrm{ppm})$ 168.7, 167.3, 79.4, 73.4, 62.0, 61.7, 57.7, 54.8, 24.6, 13.95, 13.91, 3.6. IR (neat): 2985, 1741, 1447, 1370, 1210, 1033, $863 \mathrm{~cm}^{-1}$. HRMS(EI): calcd for $\mathrm{C}_{18} \mathrm{H}_{26} \mathrm{O}_{8}\left(\mathrm{M}^{+}\right)$370.1628, obsd 370.1627.

To a stirred suspension of NaH (77.8 mg, $3.2 \mathrm{mmol})$ and dry THF (40 mL) in a $100 \mathrm{~mL}$ RBF, 2,3,3-trisethoxycarbonyl-hept-5-ynoic acid ethyl ester $(1 \mathrm{~g}, 2.7 \mathrm{mmol})$ was added in two portions under $\mathrm{N}_{2}$ counter-flow. The resulting solution was stirred at room temperature for $1 \mathrm{~h}$ after which time 1-bromo-2-butyne (490 mg, 4.1 mmol) was added. The reaction mixture was then heated at reflux for $44 \mathrm{hrs}$. The solution was cooled to room temperature and quenched with $20 \mathrm{~mL}$ sat. $\mathrm{NH}_{4} \mathrm{Cl}$ solution. The aqueous layer was extracted $3 \times 10 \mathrm{~mL} \mathrm{Et} 2 \mathrm{O}$. The collected organics were washed with brine, dried with anhydrous $\mathrm{Na}_{2} \mathrm{SO}_{4}$, and concentrated in vacuo. The crude product was purified by column chromatography eluting with 15\% EtOAc/hexanes producing a yellow oil which was further purified by triturating with hexanes to yield 9 (982.3 mg, 89\%) as a white solid. ${ }^{1} \mathrm{H}$ NMR (300 MHz, $\left.\mathrm{CDCl}_{3}\right): \delta(\mathrm{ppm}) 6.07-5.90(\mathrm{~m}, 1 \mathrm{H}), 5.11$ (d, $\left.J=17.0 \mathrm{~Hz}, 1 \mathrm{H}\right), 5.05$ (d, $\left.J=10.1 \mathrm{~Hz}, 1 \mathrm{H}\right), 4.35-4.10(\mathrm{~m}, 8 \mathrm{H}), 3.07$ (q, $J=2.4 \mathrm{~Hz}, 2 \mathrm{H}), 2.86$ (d, $J=7.2 \mathrm{~Hz}, 2 \mathrm{H}), 1.75$ (t, $2.4 \mathrm{~Hz}, 3 \mathrm{H}), 1.28$ (appt. q, $12 \mathrm{H}) .{ }^{13} \mathrm{C}\left\{{ }^{1} \mathrm{H}\right\} \mathrm{NMR}(75 \mathrm{MHz}$, $\left.\mathrm{CDCl}_{3}\right): \delta(\mathrm{ppm}) 169.2,161.1,134.0,119.1,78.1,74.8,62.6,62.3,61.9,61.7,36.3,22.8,14.01,13.99,3.8 . \quad$ IR (neat): 2982, 1731, 1367, 1330, 1211, $1035 \mathrm{~cm}^{-1}$. HRMS(CI): calcd for $\mathrm{C}_{21} \mathrm{H}_{31} \mathrm{O}_{8}\left(\mathrm{MH}^{+}\right)$411.2019, obsd 411.1999.

General [2+2+2] Cycloaddition Procedure with aldehydes. In a glove box, the diyne and aldehyde were added to an oven dried scintillation vial equipped with a magnetic stir bar and dissolved in toluene. To the stirring solution, a solution of $\mathrm{Ni}(\mathrm{COD})_{2}$ and $\operatorname{SIPr}$ (previously equilibrated for at least $\left.6 \mathrm{~h}\right)^{7}$ was added. The reaction mixture was stirred at room temperature for $2 \mathrm{~h}$ (or until complete consumption of starting material was observed as 
judged by GC or TLC) over which time the color changed from orange to brown. The mixture was concentrated in vacuo and purified by silica gel column chromatography.

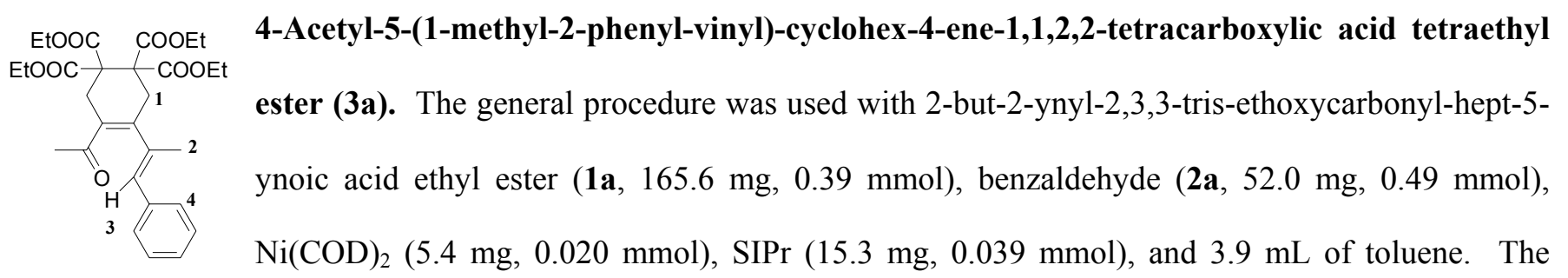
reaction mixture was purified by flash chromatography (12\% EtOAc/hexanes then $15 \%$ EtOAc/hexanes) to yield dienone 3a (166.3 mg, 80\%) as a sticky pale yellow oil. ${ }^{1} \mathrm{H}$ NMR (300 MHz, $\left.\mathrm{CDCl}_{3}\right): \delta(\mathrm{ppm}) 7.36-7.29(\mathrm{~m}, 2 \mathrm{H})$, 7.25-7.19 (m, 3H), 6.24 (br s, 1H), 4.30-4.14 (brm, 8H), 3.12 (s, 2H), 3.07 (s, 2H), 2.20 (s, 3H), 2.10 (brs, 3H), 1.27 (t, $J=7.2 \mathrm{~Hz}, 12 \mathrm{H}) .{ }^{13} \mathrm{C}\left\{{ }^{1} \mathrm{H}\right\} \mathrm{NMR}\left(75 \mathrm{MHz}, \mathrm{CDCl}_{3}\right): \delta(\mathrm{ppm}) 202.7,169.72,169.65,145.9,137.5,137.0,132.5$, $130.4,128.8,128.4,127.2,62.0,61.9,57.3,56.6,35.2,32.1,30.3,16.9,14.0$. IR (neat): 2983, 1723, 1672, 1264, 1200, 1098, $1056 \mathrm{~cm}^{-1}$. HRMS(CI): calcd for $\mathrm{C}_{29} \mathrm{H}_{36} \mathrm{O}_{9}\left(\mathrm{M}^{+}\right)$528.2359, obsd 528.2369. COSY summary; the following pertinent cross peaks were observed: $\mathrm{H}(3)$ and $\mathrm{H}(2)$ and $\mathrm{H}(4)$. NOE summary; the following pertinent enhancements were observed: irradiation of $\mathrm{H}(2)$ resulted in the enhancement of $\mathrm{H}(1)$ and $\mathrm{H}(4)$.

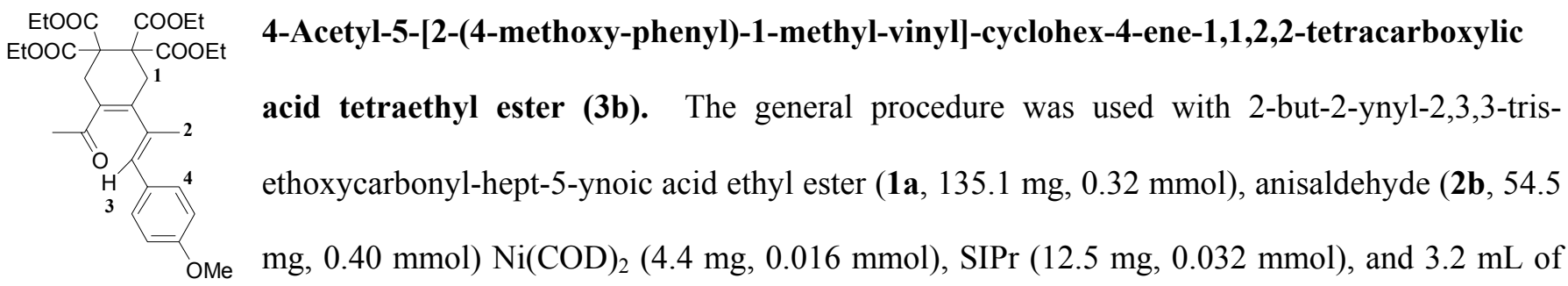
toluene. The reaction mixture was purified by flash chromatography (12\% EtOAc/hexanes then $15 \%$ EtOAc/hexanes) to yield dienone $\mathbf{3 b}(163.8 \mathrm{mg}, 92 \%)$ as a sticky pale yellow oil. ${ }^{1} \mathrm{H}$ NMR $\left(300 \mathrm{MHz}, \mathrm{CDCl}_{3}\right): \delta$ (ppm) $7.18(\mathrm{~d}, J=8.0 \mathrm{~Hz}, 2 \mathrm{H}), 6.87(\mathrm{~d}, J=8.0 \mathrm{~Hz}, 2 \mathrm{H}), 6.18$ (brs, 1H), 4.31-4.15 (brm, 8H), 3.79 (s, 3H), 3.11 (s, 2H), $3.06(\mathrm{~s}, 2 \mathrm{H}), 2.17(\mathrm{~s}, 3 \mathrm{H}), 2.06$ (brs, 3H), $1.26(\mathrm{t}, J=6.8 \mathrm{~Hz}, 12 \mathrm{H}) .{ }^{13} \mathrm{C}\left\{{ }^{1} \mathrm{H}\right\} \mathrm{NMR}\left(75 \mathrm{MHz}, \mathrm{CDCl}_{3}\right): \delta$ (ppm) 203.0, 169.82, 169.75, 158.7, 146.7, 135.7, 132.5, 130.3, 130.2, 129.7, 113.9, 62.1, 62.0, 57.5, 56.7, 55.4, 35.2, 32.1, 30.4, 16.9, 14.0. IR (neat): 2983, 1738, 1670, 1607, 1260, $1036 \mathrm{~cm}^{-1}$. HRMS(EI): calcd for $\mathrm{C}_{30} \mathrm{H}_{38} \mathrm{O}_{10}$ $\left(\mathrm{M}^{+}\right)$558.2465, obsd 558.2471. COSY summary; the following pertinent cross peaks were observed: $\mathrm{H}(3)$ and 
$\mathrm{H}(2)$ and $\mathrm{H}(4)$. NOE summary; the following pertinent enhancements were observed: irradiation of $\mathrm{H}(2)$ resulted in the enhancement of $\mathrm{H}(1)$ and $\mathrm{H}(4)$.

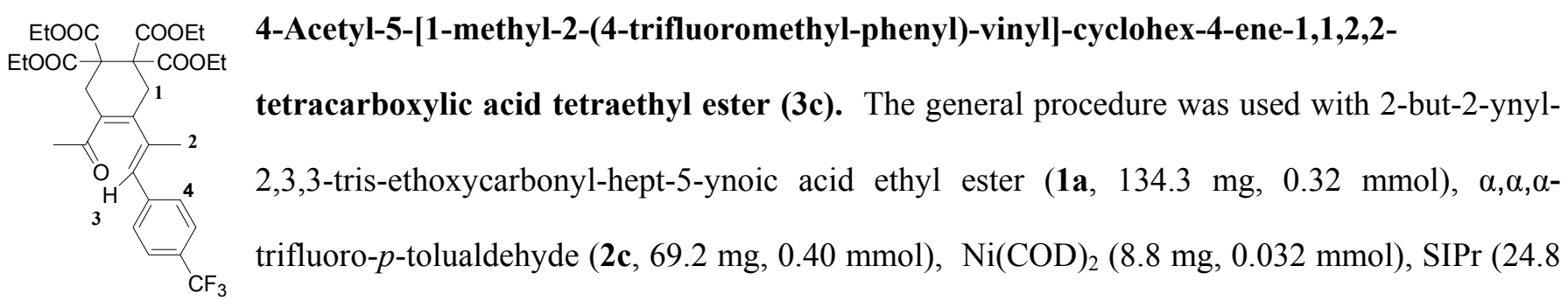

$\mathrm{mg}, 0.064 \mathrm{mmol})$, and $3.2 \mathrm{~mL}$ of toluene. The reaction mixture was purified by flash chromatography $(12 \%$ EtOAc/hexanes then $15 \%$ EtOAc/hexanes) to yield dienone $3 \mathrm{c}(127.1 \mathrm{mg}, 67 \%)$ as a sticky yellow oil. ${ }^{1} \mathrm{H}$ NMR $\left(300 \mathrm{MHz}, \mathrm{CDCl}_{3}\right): \delta(\mathrm{ppm}) 7.60(\mathrm{~d}, J=8.1 \mathrm{~Hz}, 2 \mathrm{H}), 7.35$ (d, $\left.J=8.1 \mathrm{~Hz}, 2 \mathrm{H}\right), 6.27$ (brs, 1H), 4.33-4.17 (brm, 8H), 3.12 (brs, 2H), 3.08 (brs, 2H), $2.21(\mathrm{~s}, 3 \mathrm{H}), 2.07(\mathrm{~d}, J=1.2 \mathrm{~Hz}, 3 \mathrm{H}), 1.29(\mathrm{t}, J=7.1 \mathrm{~Hz}, 12 \mathrm{H}) .{ }^{13} \mathrm{C}\left\{{ }^{1} \mathrm{H}\right\} \mathrm{NMR}(75$ $\left.\mathrm{MHz}, \mathrm{CDCl}_{3}\right): \delta(\mathrm{ppm}) 204.4,169.8,169.7,145.2,140.7,140.2,132.7,129.1,128.6,125.46,125.42,62.2,62.1$, 57.4, 56.6, 35.4, 32.2, 30.3, 17.1, 14.1. IR (neat): 2985, 1734, 1617, 1416, 1230, 1124, $867 \mathrm{~cm}^{-1}$. HRMS(EI): calcd for $\mathrm{C}_{30} \mathrm{H}_{35} \mathrm{~F}_{3} \mathrm{O}_{9}\left(\mathrm{M}^{+}\right)$596.2233, obsd 596.2241. COSY summary; the following pertinent cross peaks were observed: $\mathrm{H}(3)$ and $\mathrm{H}(2)$ and $\mathrm{H}(4)$. NOE summary; the following pertinent enhancements were observed: irradiation of $\mathrm{H}(2)$ resulted in the enhancement of $\mathrm{H}(1)$ and $\mathrm{H}(4)$.

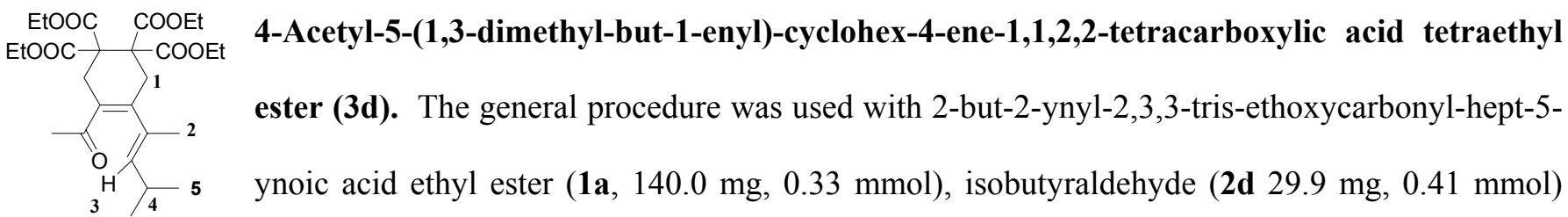
$\mathrm{Ni}(\mathrm{COD})_{2}(9.1 \mathrm{mg}, 0.033 \mathrm{mmol}), \mathrm{SIPr}(12.9 \mathrm{mg}, 0.033 \mathrm{mmol})$, and $3.3 \mathrm{~mL}$ of toluene. The reaction mixture was purified by flash chromatography $(12 \%$ EtOAc/hexanes then $15 \%$ EtOAc/hexanes $)$ collecting the fractions containing the spot with Rf 0.28 in $30 \%$ EtOAc/hexanes (purple with anisaldehyde stain). The remaining mixed fractions containing the dienolic ether and impurities were combined and allowed to re-equilibrate for 4 hrs. The re-equilibrated mixture was then re-chromatographed (12\% EtOAc/hexanes then $15 \%$ EtOAc/hexanes). This process was repeated twice to yield dienone $3 \mathbf{d}$ and a small amount of the dienolic ether $(98.5 \mathrm{mg}, 60 \%)$ as a sticky yellow oil. ${ }^{1} \mathrm{H}$ NMR (300 MHz, $\mathrm{CDCl}_{3}$ ): $\delta(\mathrm{ppm}) 5.04$ (dq, $\left.J=9.1,1.1 \mathrm{~Hz}, 1 \mathrm{H}\right), 4.32-4.12$ (brm, 8H), 3.00 (s, 4H), $2.52(\operatorname{septd}, J=6.6,2.4 \mathrm{~Hz}, 1 \mathrm{H}), 2.16(\mathrm{~s}, 3 \mathrm{H}), 1.82(\mathrm{~d}, J=1.1 \mathrm{~Hz}, 3 \mathrm{H}), 1.27$ (appt td, $J=7.1,1.7 \mathrm{~Hz}, 12 \mathrm{H}), 0.94$ 
$(\mathrm{d}, \mathrm{J}=6.7 \mathrm{~Hz}, 6 \mathrm{H}) .{ }^{13} \mathrm{C}\left\{{ }^{1} \mathrm{H}\right\} \mathrm{NMR}\left(75 \mathrm{MHz}, \mathrm{CDCl}_{3}\right): \delta(\mathrm{ppm}) 203.4,169.9,169.8,145.8,138.8,132.9,131.8$, $62.1,61.9,57.4,56.7,35.3,32.0,30.5,27.5,22.1,15.1,14.0$. IR (neat): 2963, 1742, 1676, 1265, 1096, $1052 \mathrm{~cm}^{-1}$. HRMS(CI): calcd for $\mathrm{C}_{26} \mathrm{H}_{38} \mathrm{O}_{9}\left(\mathrm{M}^{+}\right)$494.2516, obsd 494.2509. COSY summary; the following pertinent cross peaks were observed: $\mathrm{H}(3)$ and $\mathrm{H}(2)$ and $\mathrm{H}(4) ; \mathrm{H}(4)$ and $\mathrm{H}(3)$ and $\mathrm{H}(5)$. NOE summary; the following pertinent enhancements were observed: irradiation of $\mathrm{H}(2)$ resulted in the enhancement of $\mathrm{H}(1)$ and $\mathrm{H}(4)$; irradiation of $\mathrm{H}(4)$ resulted in the enhancement of $\mathrm{H}(2)$.

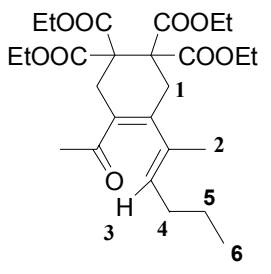

4-Acetyl-5-(1-methyl-pent-1-enyl)-cyclohex-4-ene-1,1,2,2-tetracarboxylic acid tetraethyl ester (3e). The general procedure was used with 2-but-2-ynyl-2,3,3-tris-ethoxycarbonyl-hept-5ynoic acid ethyl ester (1a, 96.7mg, $0.22 \mathrm{mmol}$ ), butyraldehyde (2e, $82.6 \mathrm{mg}, 1.15 \mathrm{mmol}$ ), $\mathrm{Ni}(\mathrm{COD})_{2}(6.3 \mathrm{mg}, 0.023 \mathrm{mmol}), \operatorname{SIPr}(9.0 \mathrm{mg}, 0.023 \mathrm{mmol})$, and $2.2 \mathrm{~mL}$ of toluene. The reaction mixture was purified by flash chromatography (12\% EtOAc/hexanes then 15\% EtOAc/hexanes) collecting the fractions containing the spot with $\mathrm{Rf} 0.28$ in $30 \%$ EtOAc/hexanes (purple with anisaldehyde stain). The remaining mixed fractions containing the dienolic ether and impurities were combined and allowed to re-equilibrate for $4 \mathrm{hrs}$. The re-equilibrated mixture was then re-chromatographed $(12 \%$ EtOAc/hexanes then $15 \%$ EtOAc/hexanes). This process was repeated twice to yield dienone $\mathbf{3 e}$ and a small amount of the dienolic ether (88.9 mg, 73\%) as a sticky pale yellow oil. ${ }^{1} \mathrm{H}$ NMR (300 $\left.\mathrm{MHz}, \mathrm{CDCl}_{3}\right): \delta(\mathrm{ppm}) 5.22(\mathrm{tq}, J=7.2,1.4 \mathrm{~Hz}, 1 \mathrm{H})$, 4.29-4.09 (brm, 8H), 2.98 (s, 4H), 2.12 (s, 3H), 1.97 (q, $J=7.3 \mathrm{~Hz}, 2 \mathrm{H}), 1.79$ (d, $J=1.3 \mathrm{~Hz}, 3 \mathrm{H}), 1.33$ (quint, $J=$ $7.4 \mathrm{~Hz}, 2 \mathrm{H}), 1.24$ (appt td, $J=7.1,1.9 \mathrm{~Hz}, 12 \mathrm{H}), 0.87(\mathrm{t}, \mathrm{J}=7.4 \mathrm{~Hz}, 3 \mathrm{H}) .{ }^{13} \mathrm{C}\left\{{ }^{1} \mathrm{H}\right\} \mathrm{NMR}\left(75 \mathrm{MHz}, \mathrm{CDCl}_{3}\right): \delta$ (ppm) 203.5, 169.9, 146.0, 135.2, 132.1, 131.9, 62.0, 61.9, 57.5, 56.7, 35.4, 32.0, 30.6, 30.4, 22.1, 15.2, 14.2, 14.1, 14.0. IR (neat): $2982,1738,1674,1445,1367,1265,1051 \mathrm{~cm}^{-1}$. HRMS(CI): calcd for $\mathrm{C}_{26} \mathrm{H}_{38} \mathrm{O}_{9}\left(\mathrm{M}^{+}\right) 494.2516$, obsd 494.2495. COSY summary; the following pertinent cross peaks were observed: $H(3)$ and $H(2)$ and $H(4)$; $\mathrm{H}(4)$ and $\mathrm{H}(3)$ and $\mathrm{H}(5)$. NOE summary; the following pertinent enhancements were observed: irradiation of $\mathrm{H}(2)$ resulted in the enhancement of $\mathrm{H}(1)$ and $\mathrm{H}(4)$; irradiation of $\mathrm{H}(4)$ resulted in the enhancement of $\mathrm{H}(2)$.

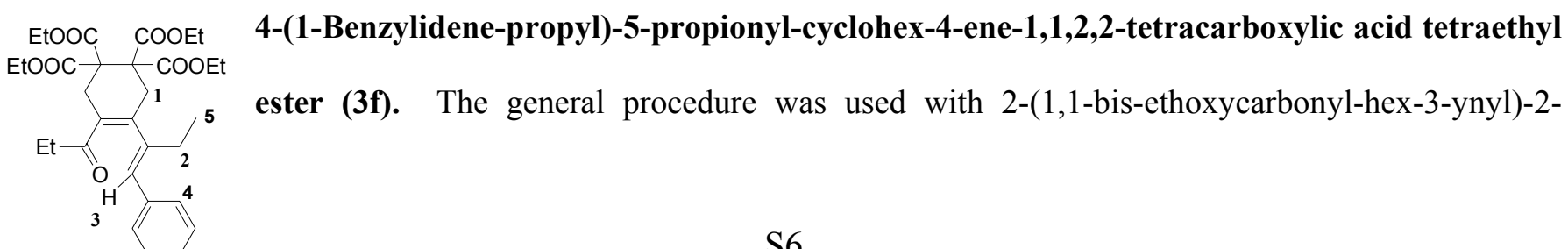


ethoxycarbonyl-hept-4-ynoic acid ethyl ester (1b, $153.3 \mathrm{mg}, 0.34 \mathrm{mmol})$, benzaldehyde (2a, $45.1 \mathrm{mg}, 43 \mathrm{mmol})$ $\mathrm{Ni}(\mathrm{COD})_{2}(13.3 \mathrm{mg}, 0.017 \mathrm{mmol}), \operatorname{SIPr}(19.8 \mathrm{mg}, 0.034 \mathrm{mmol})$, and $3.4 \mathrm{~mL}$ of toluene. The reaction mixture was purified by flash chromatography $(12 \%$ EtOAc/hexanes then $15 \%$ EtOAc/hexanes) to yield dienone $\mathbf{3 f}(158.4 \mathrm{mg}$, $84 \%$ ) as a sticky pale yellow oil. ${ }^{1} \mathrm{H}$ NMR $\left(300 \mathrm{MHz}, \mathrm{CDCl}_{3}\right): \delta(\mathrm{ppm}) 7.37-7.15(\mathrm{~m}, 5 \mathrm{H}), 6.26$ (brs, 1H), 4.314.18 (brm, 8H), 3.09 (brs, 4H), 2.59 (q, $J=7.3 \mathrm{~Hz}, 2 \mathrm{H}), 2.48$ (q, 7.6Hz, 2H), 1.29 (t, $J=7.2,12 \mathrm{H}), 1.12$ (t, $J=7.6$ $\mathrm{Hz}, 3 \mathrm{H}), 1.00(\mathrm{t}, J=7.3 \mathrm{~Hz}, 3 \mathrm{H}) .{ }^{13} \mathrm{C}\left\{{ }^{1} \mathrm{H}\right\} \mathrm{NMR}\left(75 \mathrm{MHz}, \mathrm{CDCl}_{3}\right): \delta(\mathrm{ppm}) 207.8,169.7,143.4,140.6,137.0$, 134.2, 130.0, 128.7, 128.3, 127.1, 62.0, 61.9, 57.3, 56.6, 36.2, 34.9, 33.1, 23.0, 14.0, 13.9, 12.7, 8.7. IR (neat): 2981, 2939, 1737, 1681, 1136, $1040 \mathrm{~cm}^{-1}$. HRMS(EI): calcd for $\mathrm{C}_{31} \mathrm{H}_{40} \mathrm{O}_{9}\left(\mathrm{M}^{+}\right)$556.2672, obsd 556.2681. COSY summary; the following pertinent cross peaks were observed: $\mathrm{H}(3)$ and $\mathrm{H}(2)$ and $\mathrm{H}(4)$; $\mathrm{H}(2)$ with $\mathrm{H}(5)$. NOE summary; the following pertinent enhancements were observed: irradiation of $\mathrm{H}(2)$ resulted in the enhancement of $\mathrm{H}(1)$ and $\mathrm{H}(4)$.

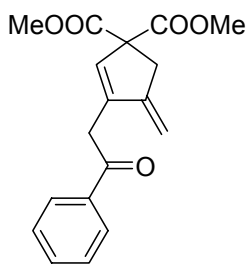

4-Methylene-3-(2-oxo-2-phenyl-ethyl)-cyclopent-2-ene-1,1-dicarboxylic acid dimethyl ester

(5a). The general procedure was used with 2,2-di-prop-2-ynyl-malonic acid dimethyl ester (4a, $118.4 \mathrm{mg}, 0.57 \mathrm{mmol})$, benzaldehyde (2a, $63.4 \mathrm{mg}, 0.60 \mathrm{mmol}), \mathrm{Ni}(\mathrm{COD})_{2}(7.8 \mathrm{mg}, 0.028$ $\mathrm{mmol}), \operatorname{SIPr}(21.8 \mathrm{mg}, 0.056 \mathrm{mmol})$, and $5.7 \mathrm{~mL}$ of toluene. The reaction mixture was purified by flash chromatography (5\% EtOAc/hexanes) to yield ketone 5a $(36.1 \mathrm{mg}, 20 \%)$ as an oily colorless solid. ${ }^{1} \mathrm{H}$ NMR $\left(300 \mathrm{MHz}, \mathrm{CDCl}_{3}\right): \delta(\mathrm{ppm}) 7.95(\mathrm{~d}, J=8.3,2 \mathrm{H}), 7.57(\mathrm{~m}, 1 \mathrm{H}), 7.46(\mathrm{~m}, 2 \mathrm{H}) 6.07(\mathrm{~s}, 1 \mathrm{H}), 4.99\left(\mathrm{td}, J_{1}=J_{2}=\right.$ $1.9 \mathrm{~Hz}, 1 \mathrm{H}), 4.95(\mathrm{t}, \mathrm{J}=2.3 \mathrm{~Hz}, 1 \mathrm{H}), 3.85(\mathrm{~d}, J=1.5,2 \mathrm{H}), 3.70(\mathrm{~s}, 6 \mathrm{H}), 3.23(\mathrm{t}, J=1.9 \mathrm{~Hz}, 2 \mathrm{H}) .{ }^{13} \mathrm{C}\left\{{ }^{1} \mathrm{H}\right\} \mathrm{NMR}$ (75 MHz, $\left.\mathrm{CDCl}_{3}\right): \delta(\mathrm{ppm}) 196.6,170.8,149.8,141.8,136.6,134.2,133.5,128.8,128.6,104.6,63.8,53.1,38.0$, 37.4. IR (neat): $3065,2955,2846,1733,1687,1434,1256 \mathrm{~cm}^{-1}$. HRMS(CI): calcd for $\mathrm{C}_{18} \mathrm{H}_{18} \mathrm{O}_{5}\left(\mathrm{M}^{+}\right) 314.1154$, obsd 315.1217 .

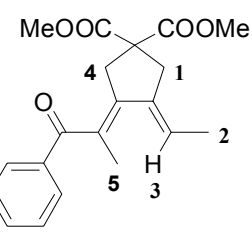

3-Ethylidene-4-(1-methyl-2-oxo-2-phenyl-ethylidene)-cyclopentane-1,1-dicarboxylic acid dimethyl ester (5b). The general procedure was used with 2,2-di-but-2-ynyl-malonic acid dimethyl ester (4b, $131.5 \mathrm{mg}, 0.56 \mathrm{mmol})$, bezaldehyde (2a, $73.8 \mathrm{mg}, 0.70 \mathrm{mmol}), \mathrm{Ni}(\mathrm{COD})_{2}$ (7.7 $\mathrm{mg}, 0.028 \mathrm{mmol}), \mathrm{S} I \operatorname{Pr}(21.8 \mathrm{mg}, 0.056 \mathrm{mmol})$, and $5.6 \mathrm{~mL}$ of toluene. The reaction mixture was purified by flash chromatography $(10 \%$ EtOAc/hexanes then $12 \%$ EtOAc/hexanes) to yield dienone $\mathbf{5 b}$ as the major isomer 
(111.6 mg, 92\%). ${ }^{1} \mathrm{H}$ NMR (500 MHz, $\left.\mathrm{CDCl}_{3}\right): \delta(\mathrm{ppm}) 7.82(\mathrm{~m}, 2 \mathrm{H}), 7.58-7.43(\mathrm{~m}, 3 \mathrm{H}), 6.01(\mathrm{q}, 6.9 \mathrm{~Hz}, 1 \mathrm{H}) 3.65$ (s, 6H), 3.04 (brs, 2H), 2.88 (brs, 2H), 2.10 (brs, 3H), 1.84 (d, $7.0 \mathrm{~Hz}, 3 \mathrm{H}) .{ }^{13} \mathrm{C}\left\{{ }^{1} \mathrm{H}\right\} \mathrm{NMR}\left(75 \mathrm{MHz}, \mathrm{CDCl}_{3}\right): \delta$ (ppm) 200.4, 171.8, 138.6, 137.9, 136.7, 133.2, 129.6, 128.9, 126.1, 57.4, 53.0, 41.1, 37.9, 18.6, 16.0. IR (neat): 2955, 2856, 1737, 1662, 1443, 1269, 1067, $972 \mathrm{~cm}^{-1}$. HRMS(EI): calcd for $\mathrm{C}_{20} \mathrm{H}_{22} \mathrm{O}_{5}\left(\mathrm{M}^{+}\right) 342.1467$, obsd 342.1468. COSY summary; the following pertinent cross peaks were observed: $H(3)$ and $H(1)$ and $H(2)$; $H(4)$ with $\mathrm{H}(5)$. NOE summary; the following pertinent enhancements were observed: irradiation of $\mathrm{H}(1)$ resulted in the enhancement of $\mathrm{H}(2)$; irradiation of $\mathrm{H}(2)$ resulted in the enhancement of $\mathrm{H}(1)$; irradiation of $\mathrm{H}(3)$ results in the enhancement of $\mathrm{H}(5)$; irradiation of $\mathrm{H}(5)$ results in the enhancement of $\mathrm{H}(3)$.
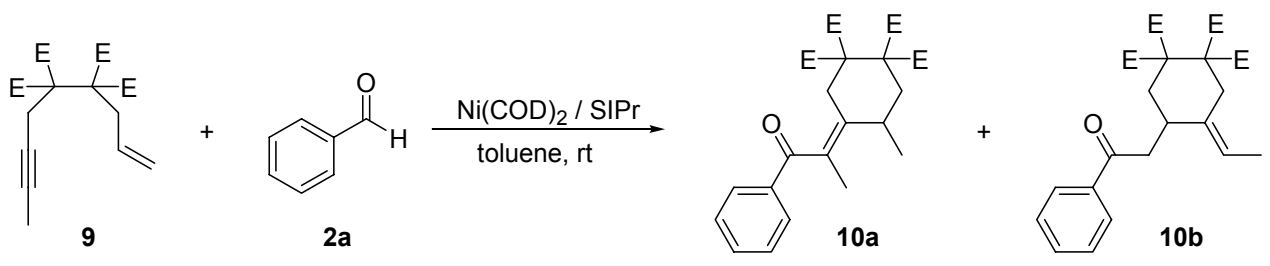

4-Methyl-5-(1-methyl-2-oxo-2-phenyl-ethylidene)-cyclohexane-1,1,2,2-tetracarboxylic acid tetraethyl ester (10a) and 4-ethylidene-5-(2-oxo-2-phenyl-ethyl)-cyclohexane-1,1,2,2-tetracarboxylic acid tetraethyl ester (10b). The general procedure was used with 2-but-2-ynyl-2,3,3-tris-ethoxycarbonyl-hept-5-ynoic acid ethyl ester (9, $130.1 \mathrm{mg}, 0.32 \mathrm{mmol})$, benzaldehyde (2a, $42.0 \mathrm{mg}, 0.40 \mathrm{mmol}), \mathrm{Ni}(\mathrm{COD})_{2}$ (4.4 mg, $\left.0.016 \mathrm{mmol}\right), \operatorname{SIPr}(12.5$ $\mathrm{mg}, 0.032 \mathrm{mmol})$, and $3.2 \mathrm{~mL}$ of toluene. The reaction mixture was purified by flash chromatography $(12 \%$ EtOAc/hexanes then $15 \%$ EtOAc/hexanes) to yield enone 10a $(46.3 \mathrm{mg}, 28 \%)$ as a sticky pale yellow oil and ketone $10 \mathrm{~b}(82.7 \mathrm{mg}, 51 \%)$ also as a sticky pale yellow oil.

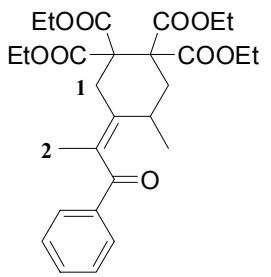

4-Methyl-5-(1-methyl-2-oxo-2-phenyl-ethylidene)-cyclohexane-1,1,2,2-tetracarboxylic acid tetraethyl ester (10a). ${ }^{1} \mathrm{H} \mathrm{NMR}\left(300 \mathrm{MHz}, \mathrm{CDCl}_{3}\right): \delta(\mathrm{ppm}) 8.11(\mathrm{~d}, J=7.7 \mathrm{~Hz}, 2 \mathrm{H}), 7.61-7.45$ $(\mathrm{m}, 3 \mathrm{H}), 4.33-4.05(\mathrm{~m}, 8 \mathrm{H}) 3.13(\mathrm{~d}, J=15.7,1 \mathrm{H}), 2.84(\mathrm{dd}, J=15.7,0.9 \mathrm{~Hz}, 1 \mathrm{H}), 2.75(\mathrm{dd}, J=$ 13.6, $6.0 \mathrm{~Hz}, 1 \mathrm{H}), 2.69$ (brt, $J=6.1 \mathrm{~Hz}, 1 \mathrm{H}), 2.27$ (dd, $J=13.7,2.6 \mathrm{~Hz}, 1 \mathrm{H}), 1.83$ (brs, 3H), 1.35-1.23 (m, 12H), $0.96(\mathrm{~d}, J=7.3 \mathrm{~Hz}, 3 \mathrm{H}) .{ }^{13} \mathrm{C}\left\{{ }^{1} \mathrm{H}\right\} \mathrm{NMR}\left(75 \mathrm{MHz}, \mathrm{CDCl}_{3}\right): \delta(\mathrm{ppm}) 201.3,170.8,170.5,170.4,168.9,136.7$, $136.3,133.4,130.9,129.8,128.9,62.1,61.8,61.7,61.5,58.7,58.4,36.0,32.8,29.1,20.5,16.1,14.03,13.99$, 13.97, 13.92. IR (neat): $2982,1735,1666,1596,1450,1266,1058 \mathrm{~cm}^{-1}$. HRMS(EI): calcd for $\mathrm{C}_{28} \mathrm{H}_{36} \mathrm{O}_{9}\left(\mathrm{M}^{+}\right)$ 
516.2359, obsd 516.2341. COSY summary; the following pertinent cross peaks were observed: $\mathrm{H}(1)$ and $\mathrm{H}(2)$. NOE summary; the following pertinent enhancements were observed: irradiation of $H(2)$ resulted in the enhancement of $\mathrm{H}(1)$; irradiation of $\mathrm{H}(1)$ resulted in the enhancement of $\mathrm{H}(2)$.

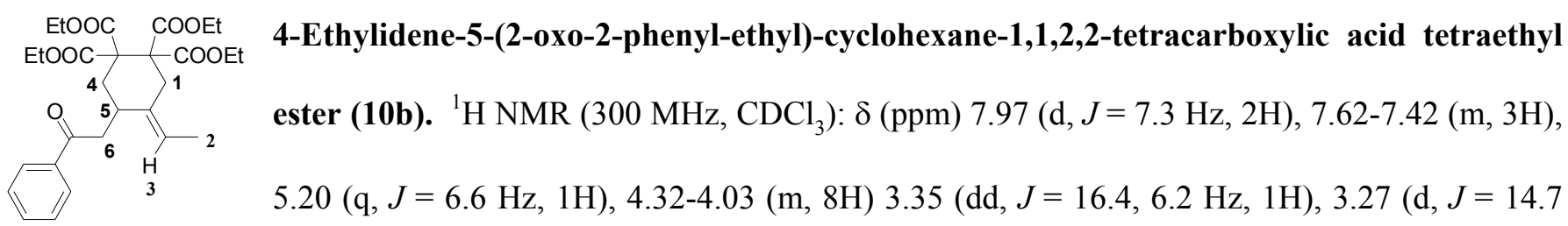

$\mathrm{Hz}, 1 \mathrm{H}), 3.13$ (brm, 1H), 2.84 (dd, $J=16.3,7.1 \mathrm{~Hz}, 1 \mathrm{H}), 2.80$ (d, $J=15.4 \mathrm{~Hz}, 1 \mathrm{H}), 2.40$ (dd, $J=13.5,4.5 \mathrm{~Hz}, 1 \mathrm{H})$, $2.20\left(\mathrm{dd}, J_{I}=J_{2}=13.0 \mathrm{~Hz}, 1 \mathrm{H}\right), 1.60(\mathrm{~d}, J=6.6 \mathrm{~Hz}, 3 \mathrm{H}), 1.30-1.18(\mathrm{~m}, 12 \mathrm{H}) .{ }^{13} \mathrm{C}\left\{{ }^{1} \mathrm{H}\right\} \mathrm{NMR}\left(75 \mathrm{MHz}, \mathrm{CDCl}_{3}\right): \delta$ (ppm) 198.8, 170.45, 170.37, 169.4, 168.9, 137.2, 134.9, 133.2, 128.7, 128.1, 117.9, 61.9, 61.62, 61.57, 59.5, 41.1, $36.3,34.6,31.9,14.0,13.92,13.86,13.2$. IR (neat): 2984, 1735, 1688, 1448, 1261, 1100, $1040 \mathrm{~cm}^{-1}$. HRMS(EI): calcd for $\mathrm{C}_{28} \mathrm{H}_{36} \mathrm{O}_{9}\left(\mathrm{M}^{+}\right)$516.2359, obsd 516.2364. COSY summary; the following pertinent cross peaks were observed: $\mathrm{H}(2)$ and $\mathrm{H}(1)$ and $\mathrm{H}(3) ; \mathrm{H}(5)$ and $\mathrm{H}(6)$ and $\mathrm{H}(7)$. NOE summary; the following pertinent enhancements were observed: irradiation of $\mathrm{H}(2)$ resulted in the enhancement of $\mathrm{H}(1)$; irradiation of $\mathrm{H}(3)$ resulted in the enhancement of $\mathrm{H}(6)$.

EtOOC COOEt
EtOOC - COOEt scintillation vial equipped with a magnetic stir bar and dissolved in $2.2 \mathrm{~mL}$ toluene. The vial was sealed with a septum and placed under $\mathrm{N}_{2}$. To the stirring solution of cyclohexanone, a solution of $\mathrm{Ni}(\mathrm{COD})_{2}(9.8 \mathrm{mg}, 0.036 \mathrm{mmol})$ and $\operatorname{SIPr}(27.7 \mathrm{mg}, 0.071 \mathrm{mmol})$ in $0.4 \mathrm{~mL}$ toluene (previously equilibrated for at least $6 \mathrm{~h}$ ) was added. A solution of 2-but-2-ynyl-2,3,3-tris-ethoxycarbonyl-hept-5-ynoic acid ethyl ester (1a, $150.0 \mathrm{mg}, 0.36 \mathrm{mmol}$ ) in $0.9 \mathrm{~mL}$ toluene was then added via syringe pump over $30 \mathrm{~min}$. The resulting brownish orange solution was stirred at room temperature for an addition $1.5 \mathrm{hrs}$. The crude reaction mixture was concentrated in vacuo and purified by flash column chromatography on $\mathrm{SiO}_{2}(12 \% \mathrm{EtOAc} /$ hexanes then $15 \%$ EtOAc/hexanes). The fractions containing both the dienolic ether (upper red spot, anisaldehyde stain) and dienone (lower purple spot, anisaldehyde stain) were combined to yield dienolic ether $\mathbf{1 2}$ and a small amount dienone (144.8 mg, 83\%) as a white solid. ${ }^{1} \mathrm{H}$ NMR $\left(300 \mathrm{MHz}, \mathrm{CDCl}_{3}\right): \delta(\mathrm{ppm}) 4.27-4.13(\mathrm{~m}, 8 \mathrm{H}), 3.01(\mathrm{~s}, 2 \mathrm{H}), 2.88(\mathrm{~s}$, 2H), $1.92(\mathrm{brd}, J=13.0 \mathrm{~Hz}, 2 \mathrm{H}), 1.86(\mathrm{~s}, 3 \mathrm{H}), 1.60(\mathrm{~s}, 3 \mathrm{H}), 1.70-1.56$ (brm, 3H), 1.51-1.40 (brm, 3H), 1.36-1.20 
(m, 14H). ${ }^{13} \mathrm{C}\left\{{ }^{1} \mathrm{H}\right\} \operatorname{NMR}\left(75 \mathrm{MHz}, \mathrm{CDCl}_{3}\right): \delta(\mathrm{ppm}) 170.2,170.1,143.9,124.0,121.8,104.9,77.7,61.8,61.7$, $58.7,57.9,31.7,31.1,30.3,25.8,21.4,16.1,14.0,12.5$. IR (neat): 2934, 2960, 1740, 1662, 1446, 1266, $1408 \mathrm{~cm}^{-1}$. HRMS(EI): calcd for $\mathrm{C}_{28} \mathrm{H}_{40} \mathrm{O}_{9}\left(\mathrm{M}^{+}\right)$520.2672, obsd 520.2673.

\section{References:}

(1) Böhm, V. P. W., Gstöttmayr, C. W. K., Weskamp, T.; Herrmann, W. A. Angew. Chem. Int. Ed. 2001, 40, $3387-3389$.

(2) Jensen, D. R.; Sigman, M. S. Org. Lett. 2003, 5, 63.

(3) Arnold, P. L.; Cloke, G. N.; Geldbach, T.; Hitchcock, P. B. Organometallics 1999, 18, 3228.

(4) Atkinson, R. S.; Grimshire, M. J. J. Chem. Soc. Perkin Trans. 1 1986, 1215.

(5) Wang, X.; Chakrapani, H.; Mandin, J. W.; Keyerleber, M. A.; Widenhofer, R. A. J. Org. Chem. 2002, 67, 2778.

(6) Liu, C.; Widenhofer, R. A. Organometallics 2002, 21, 5666.

(7) An equilibrium exists between $\mathrm{Ni}(\mathrm{COD})_{2} / \mathrm{IPr}$ and $\mathrm{Ni}(\mathrm{IPr})_{2} / \mathrm{COD}$, see: Louie, J.; Gibby, J. E.; Farnworth, M. V.; Tekavec, T. N. J. Am. Chem. Soc. 2002, 124, 15188. 


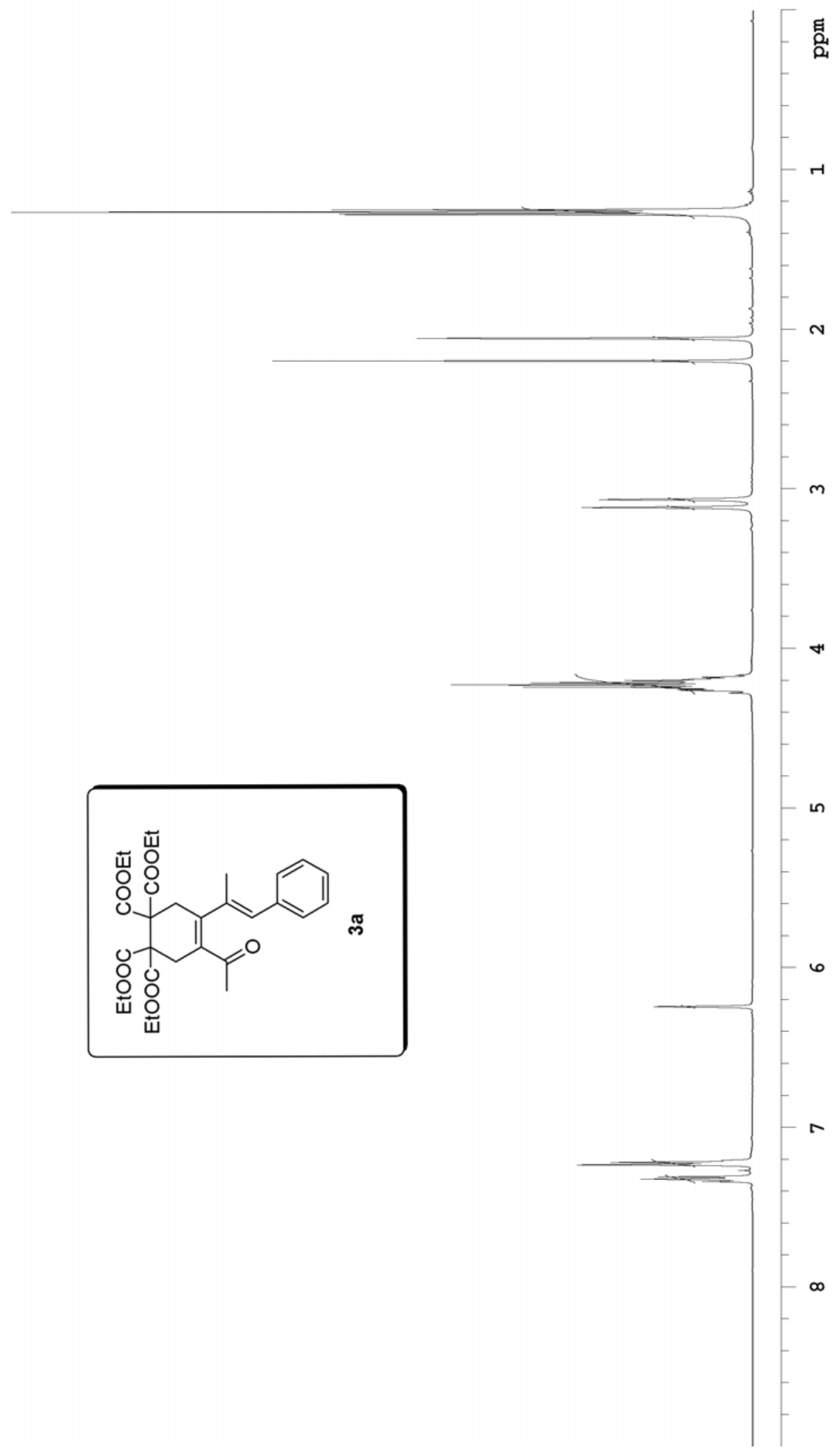




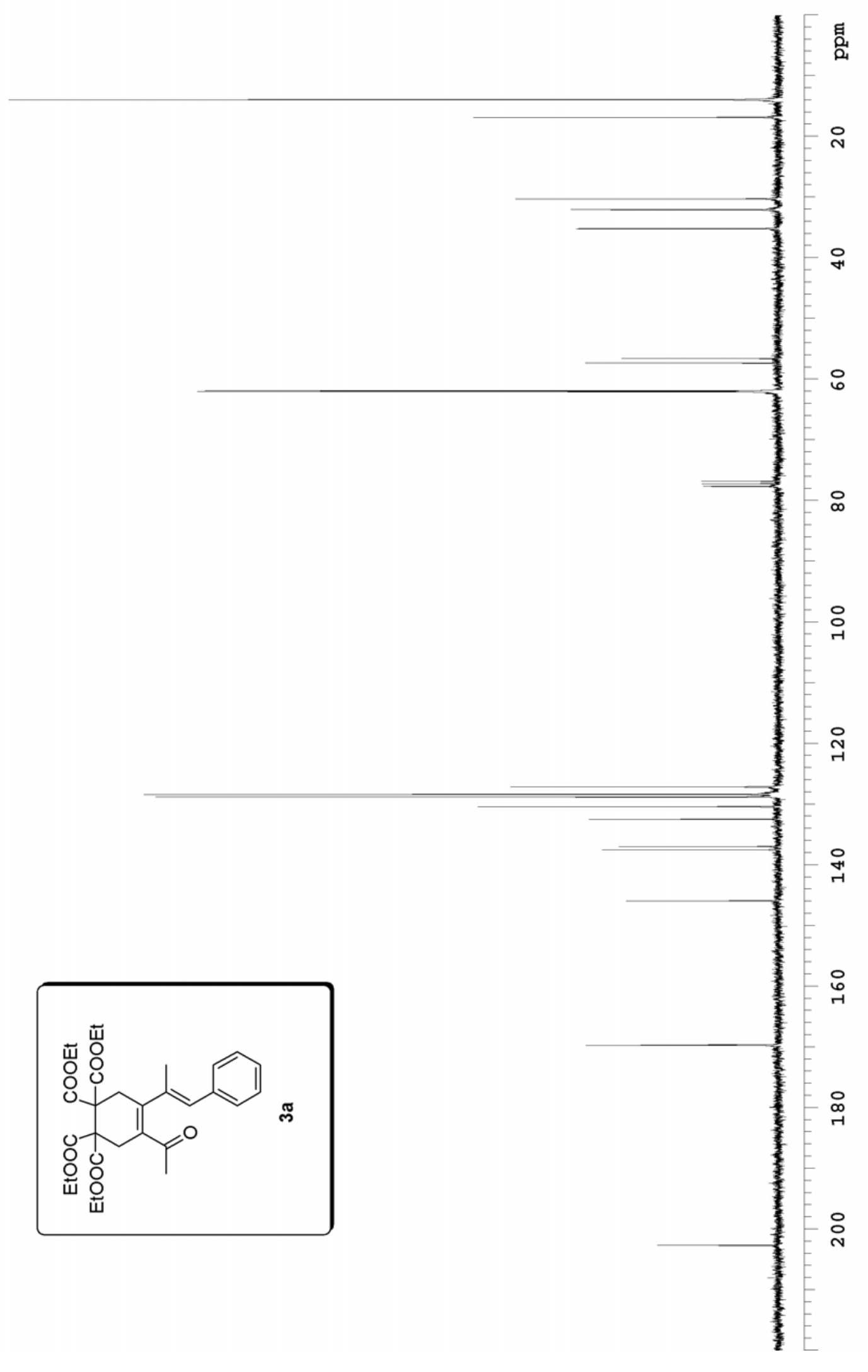



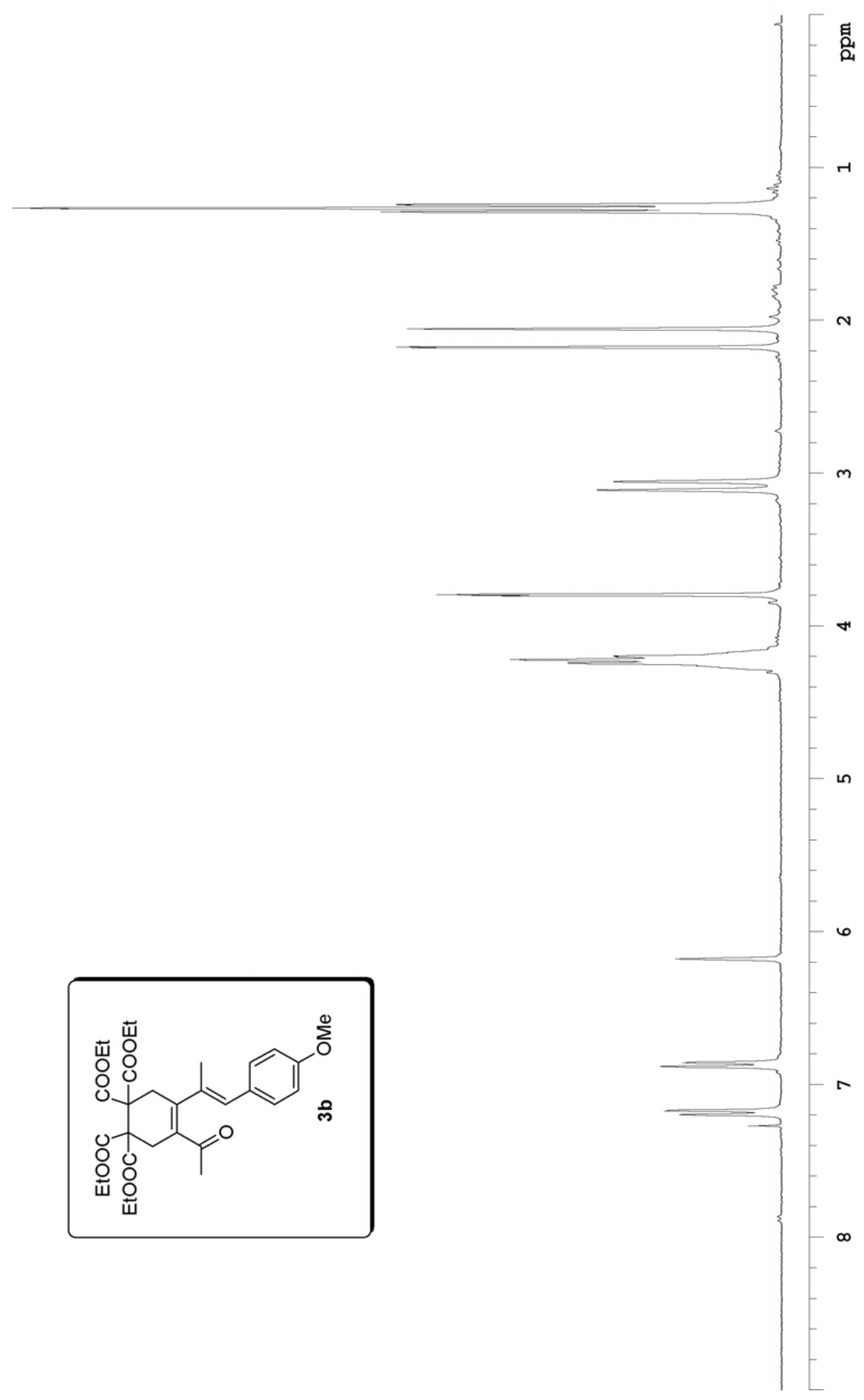


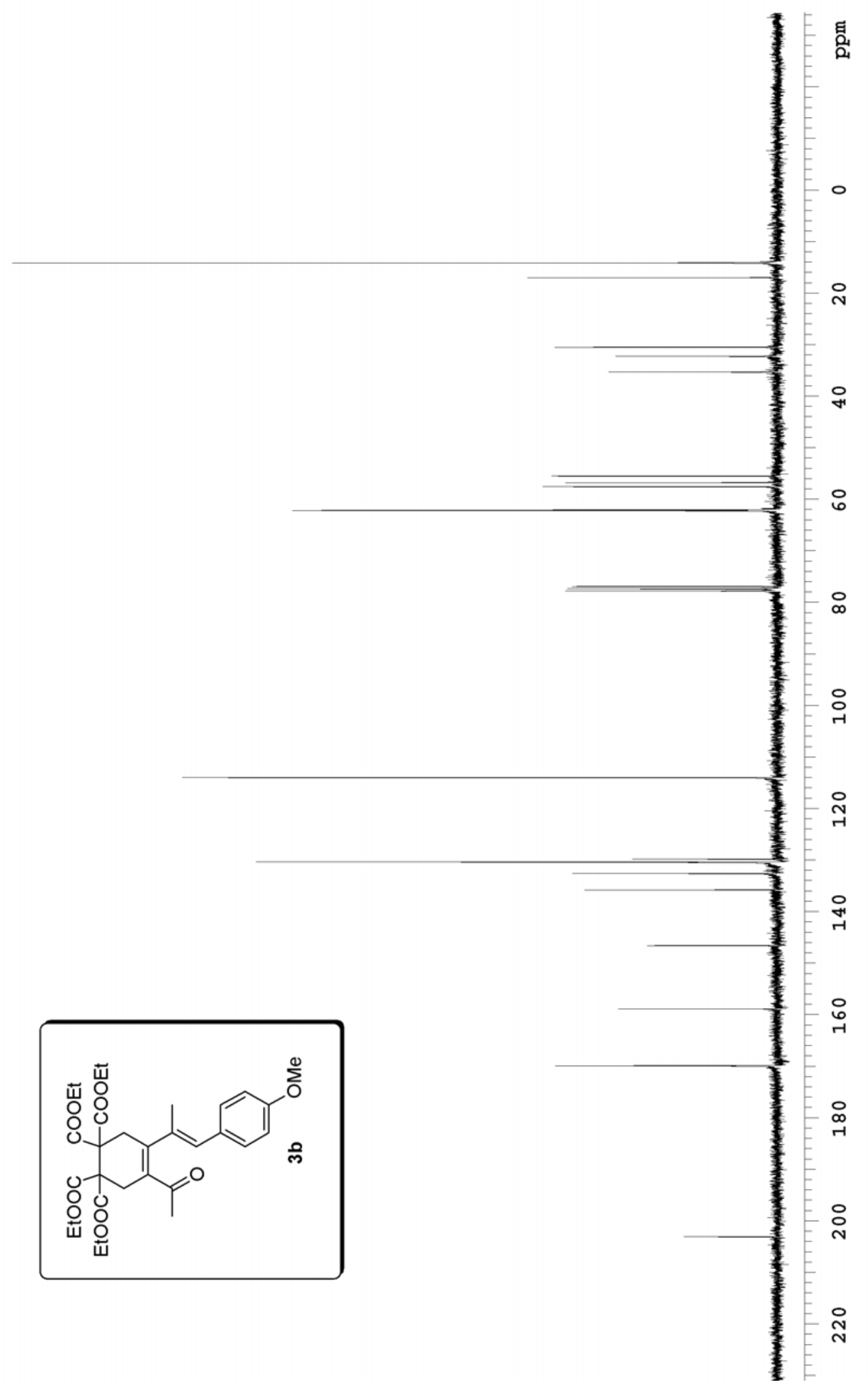




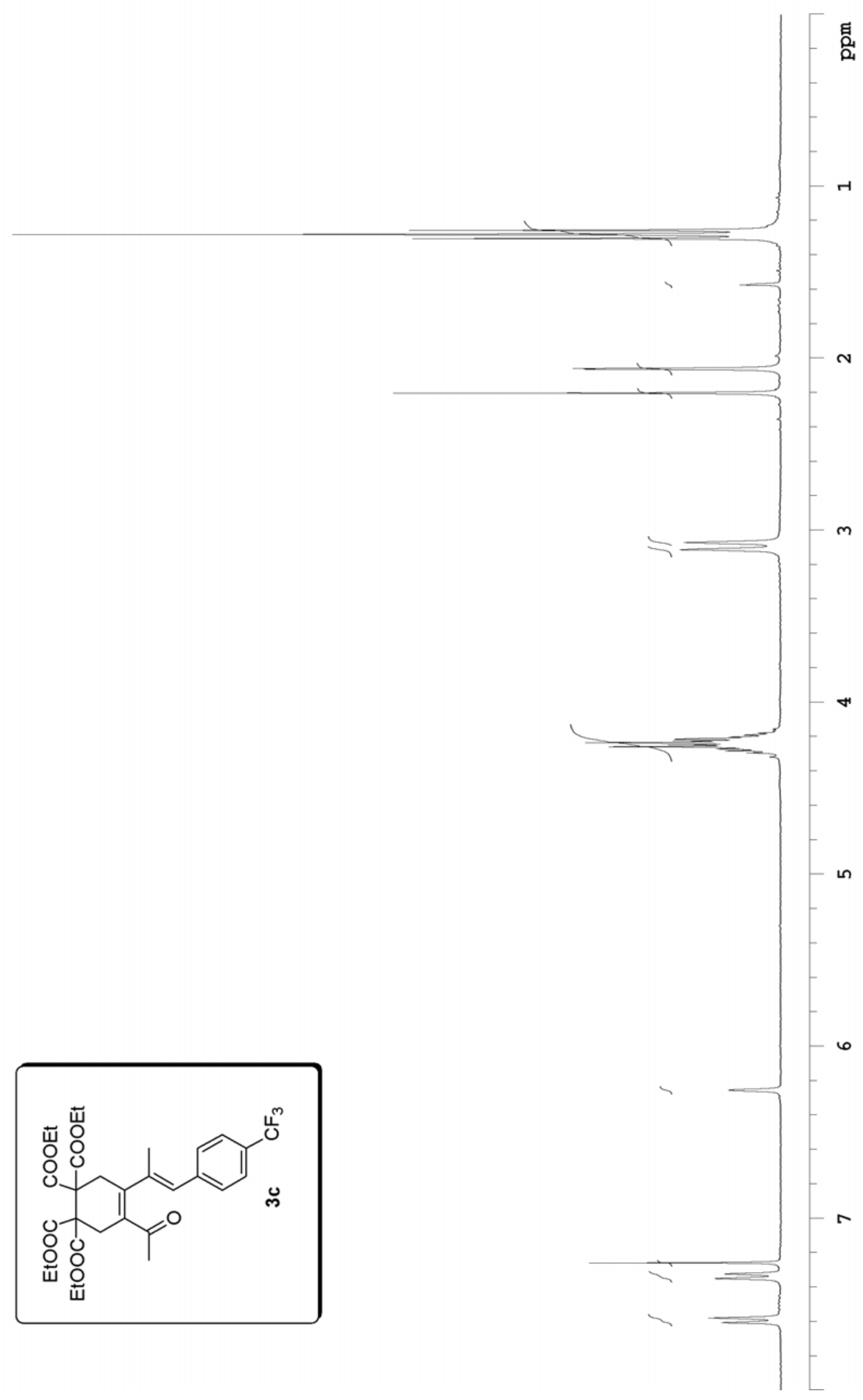




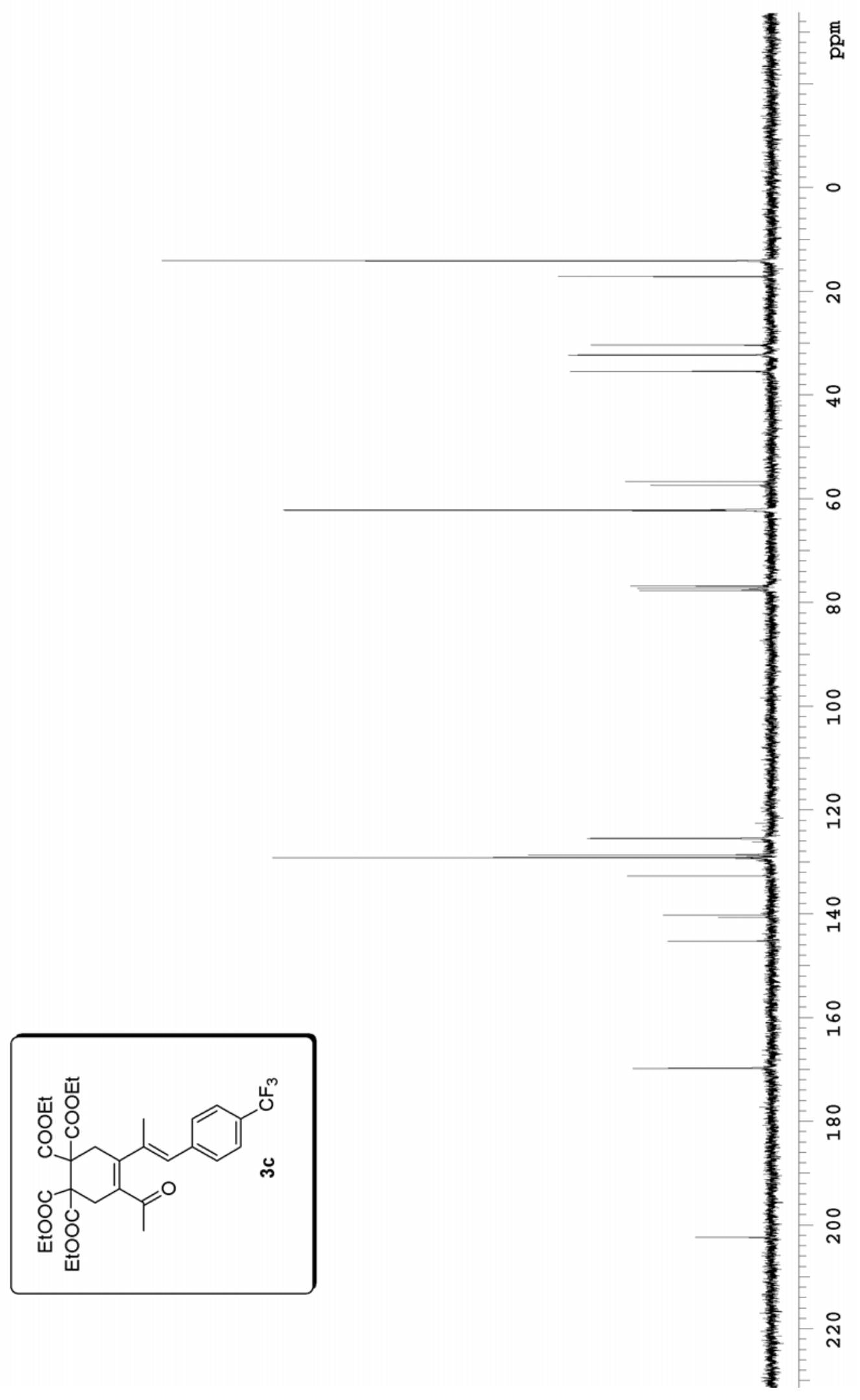

S16 


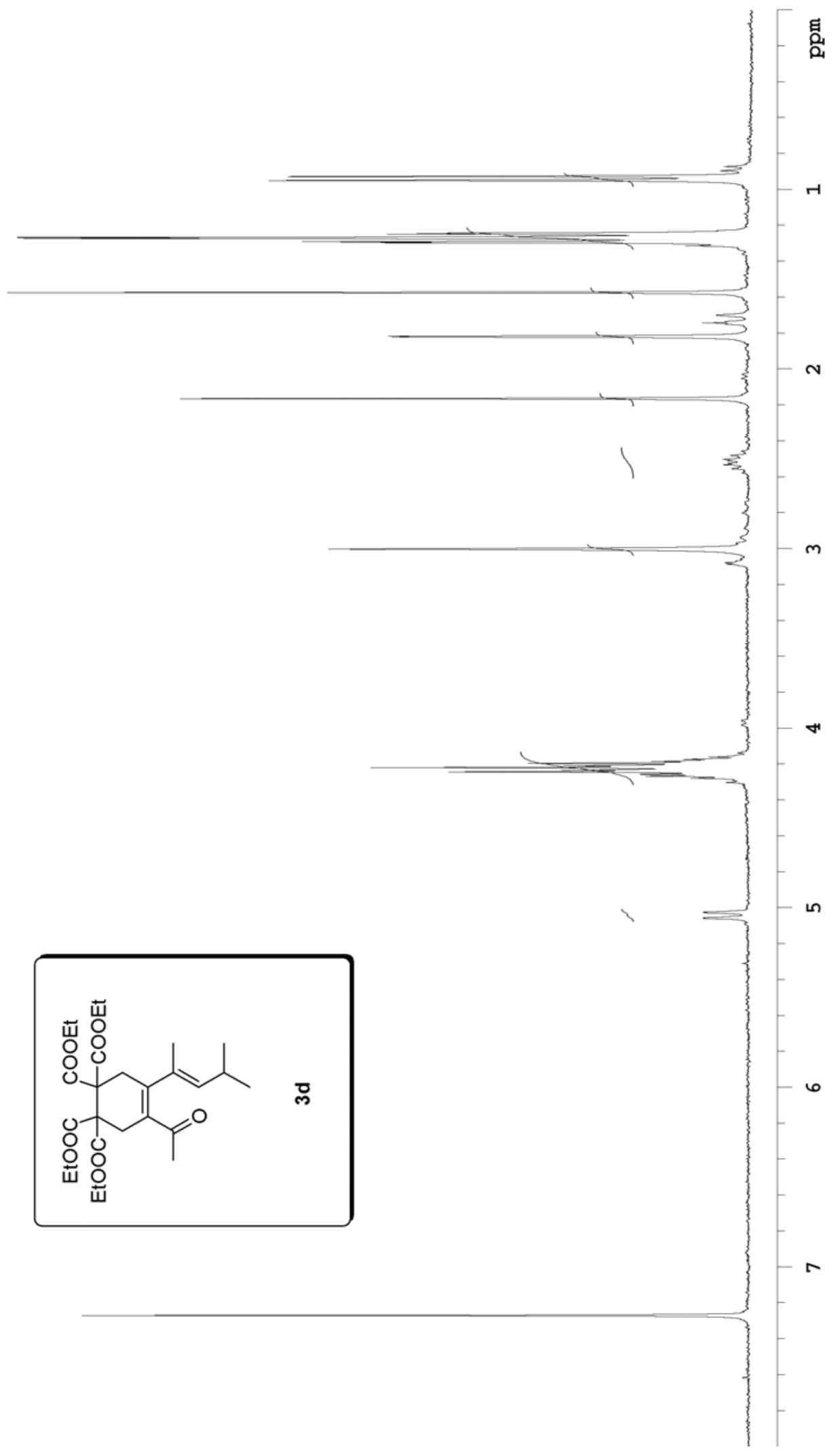




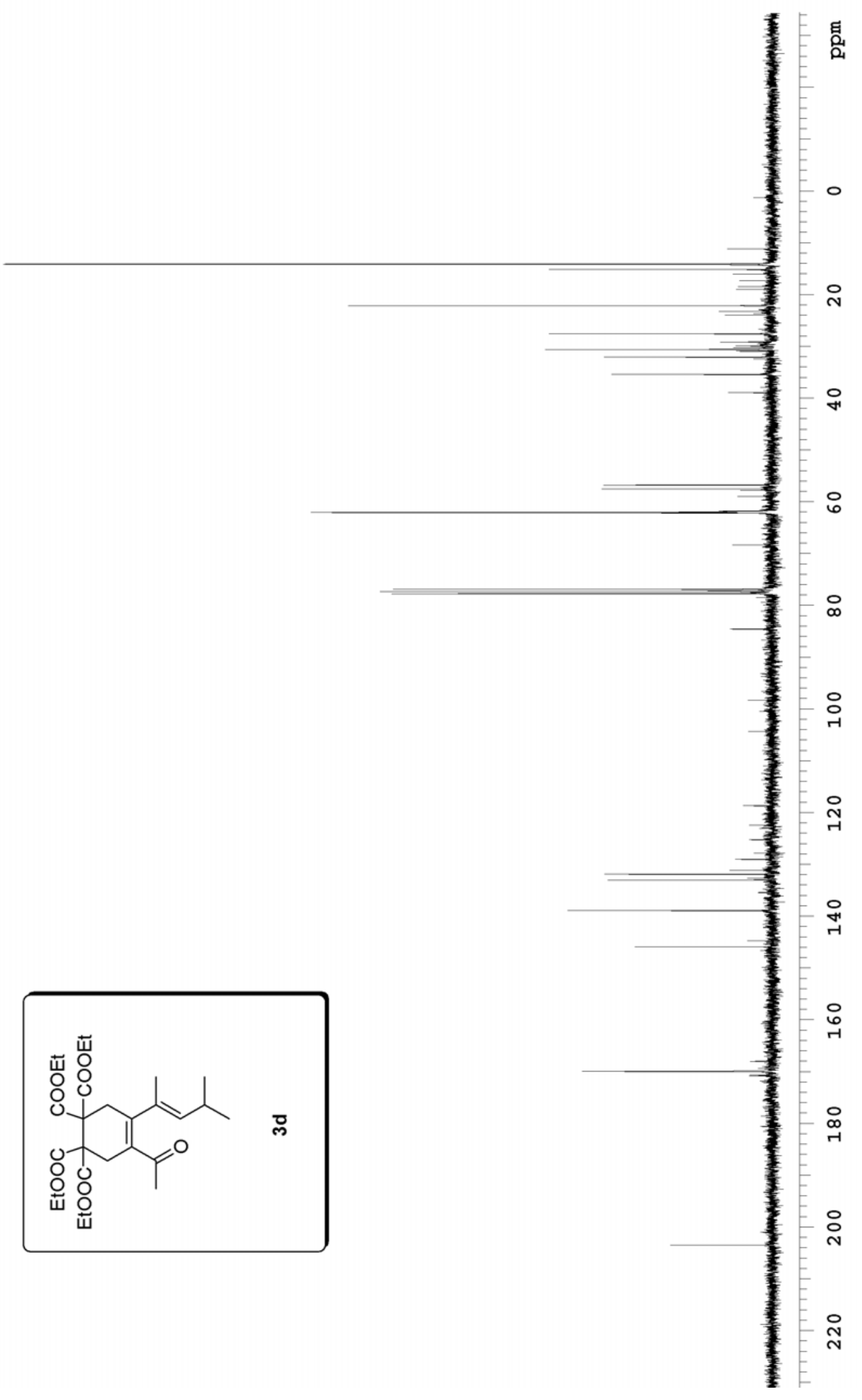




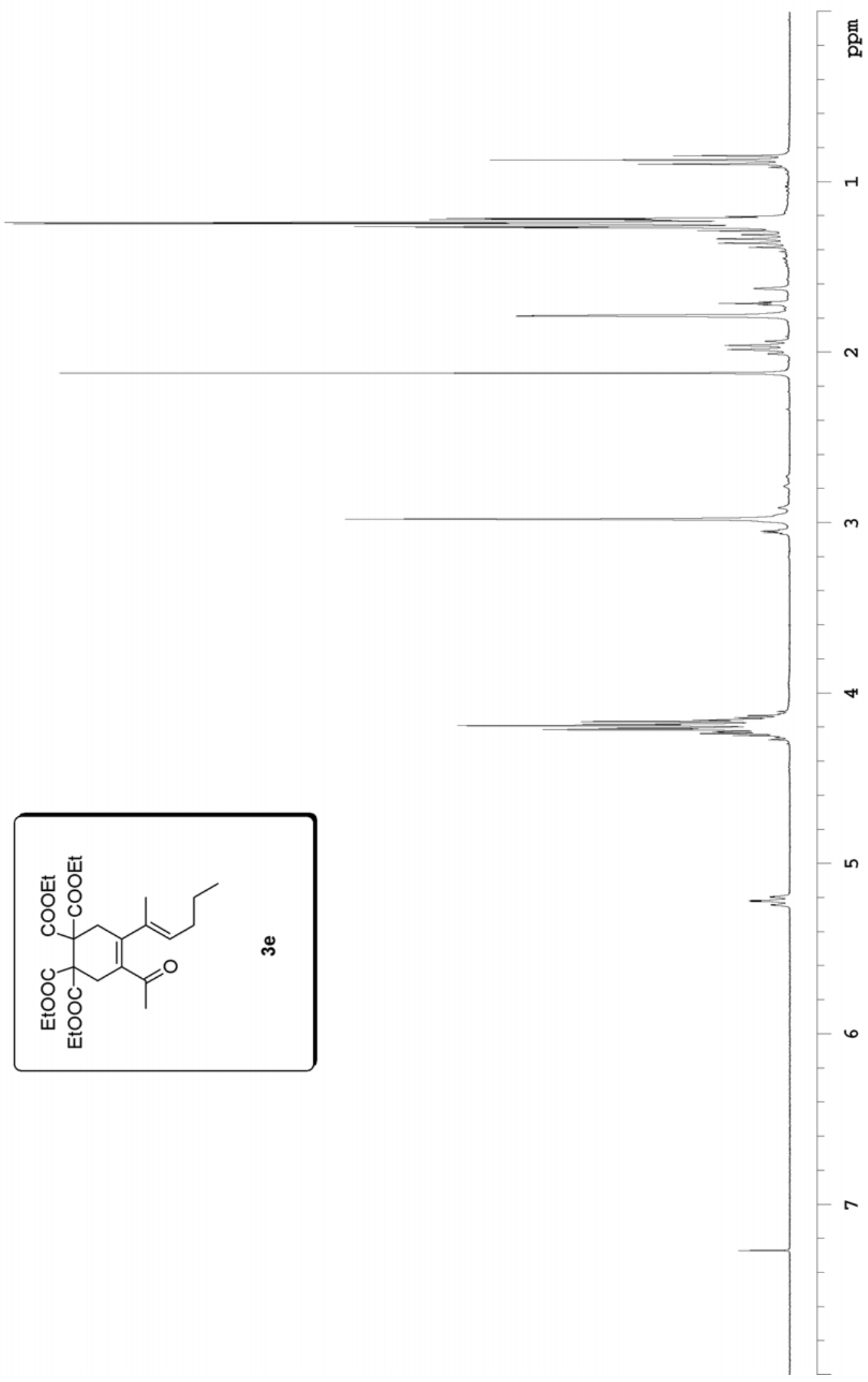




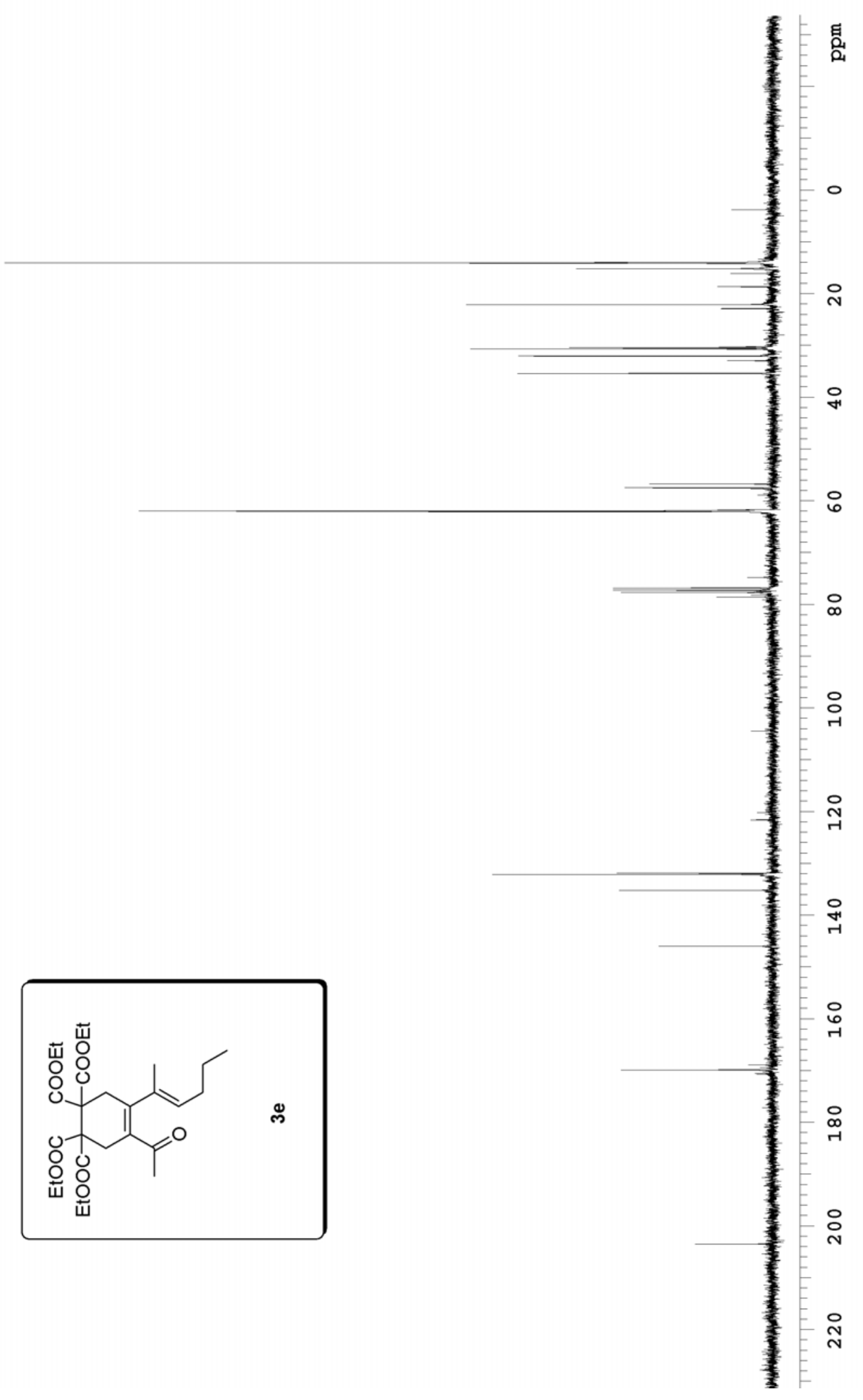




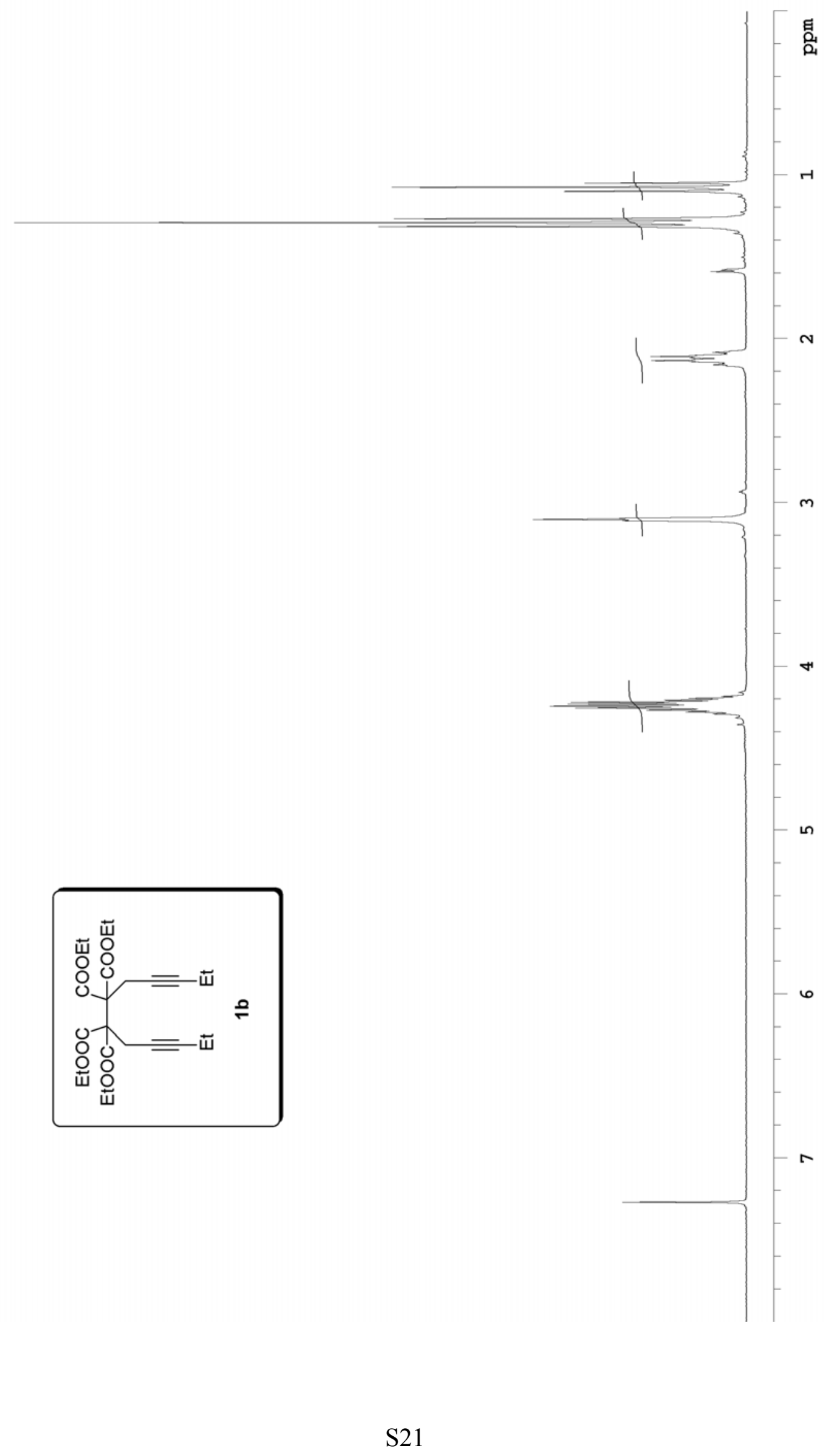



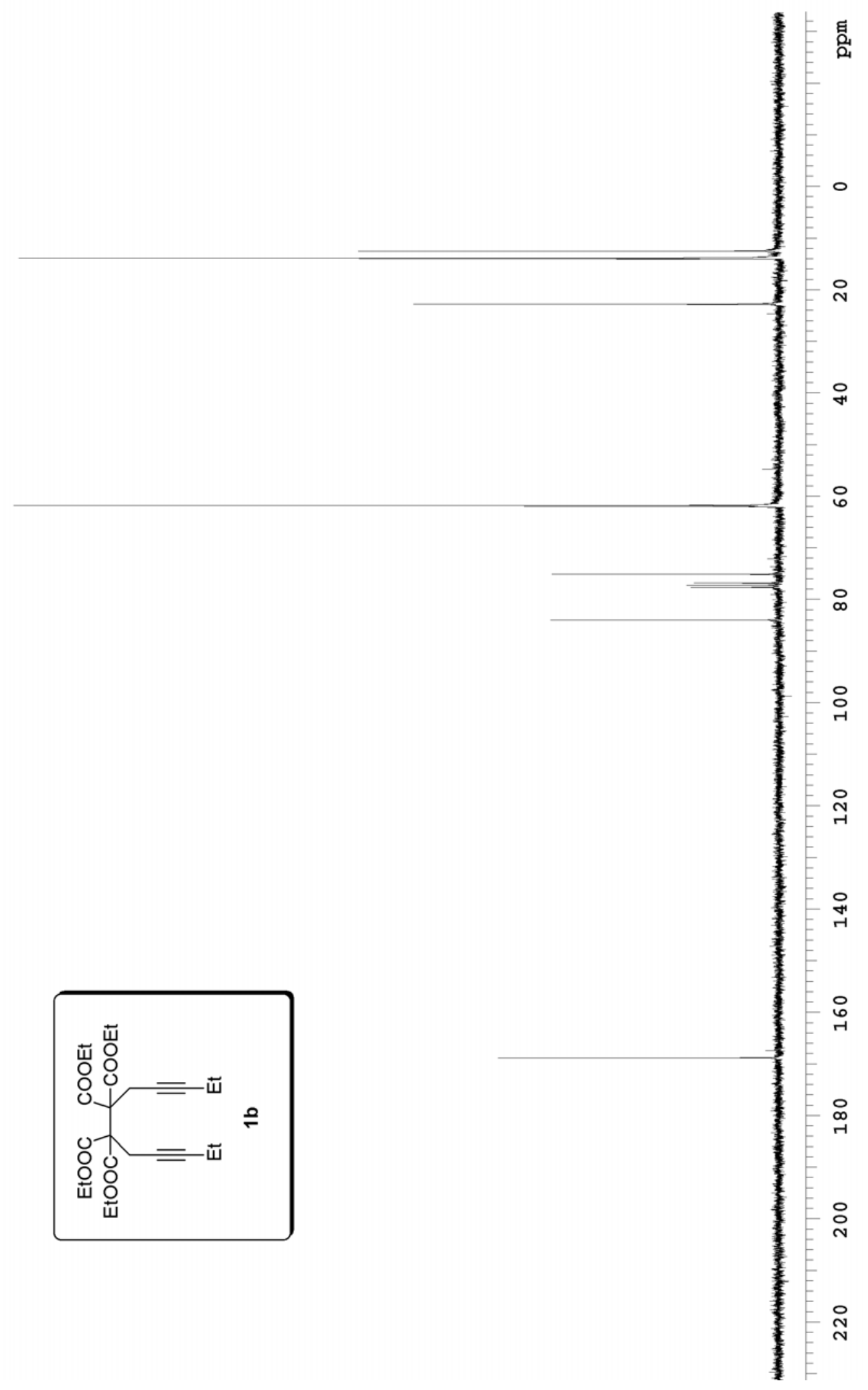


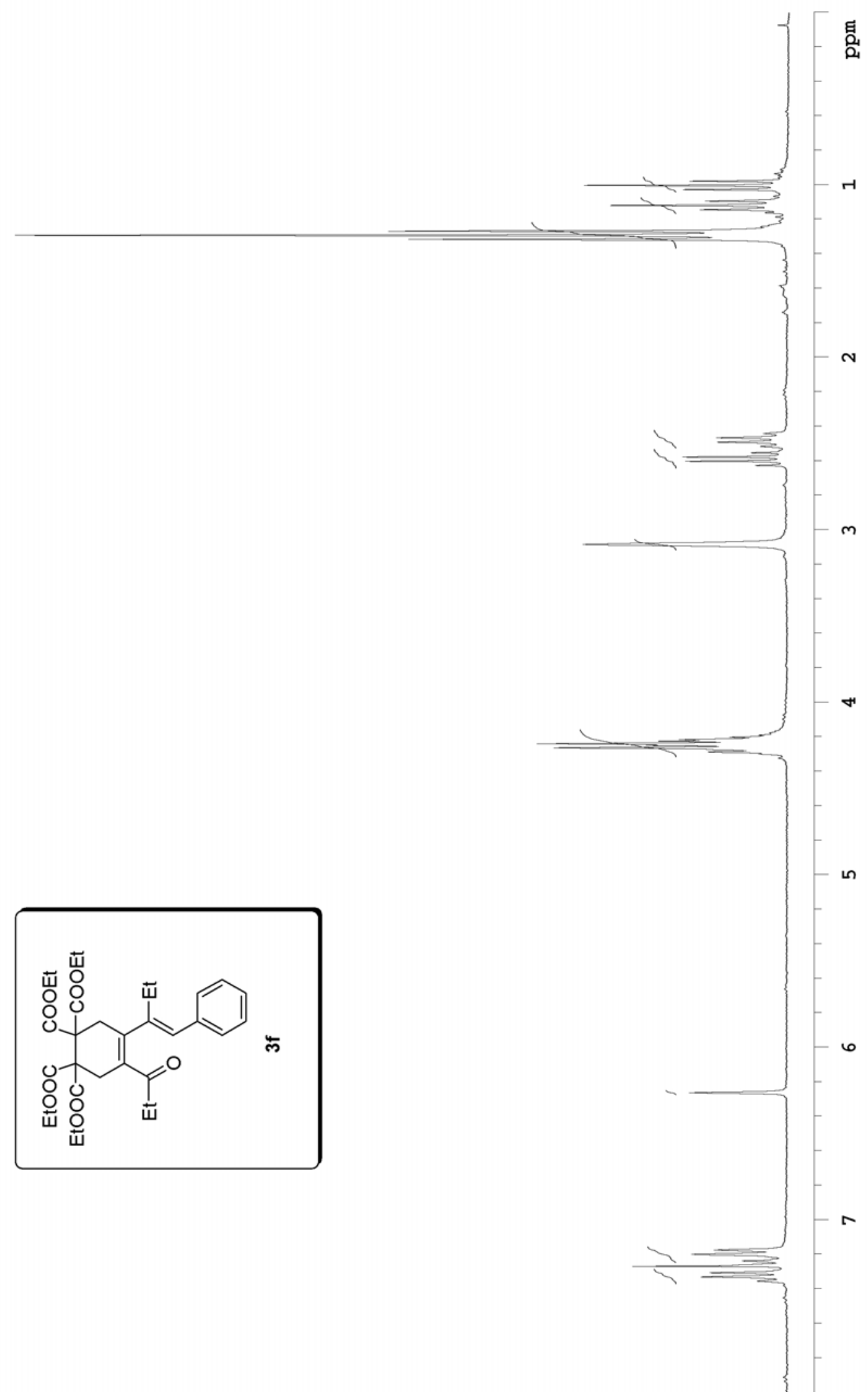




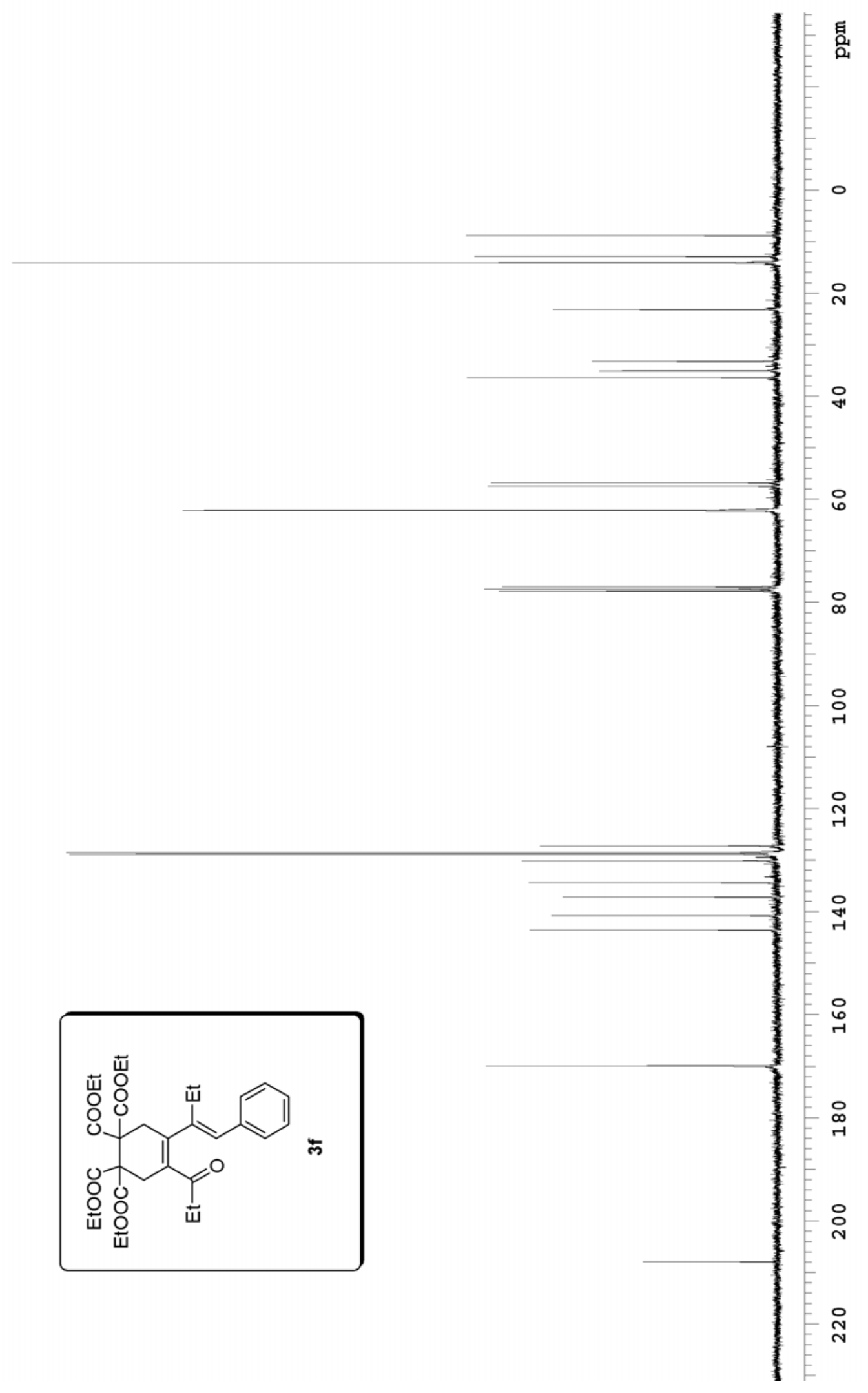




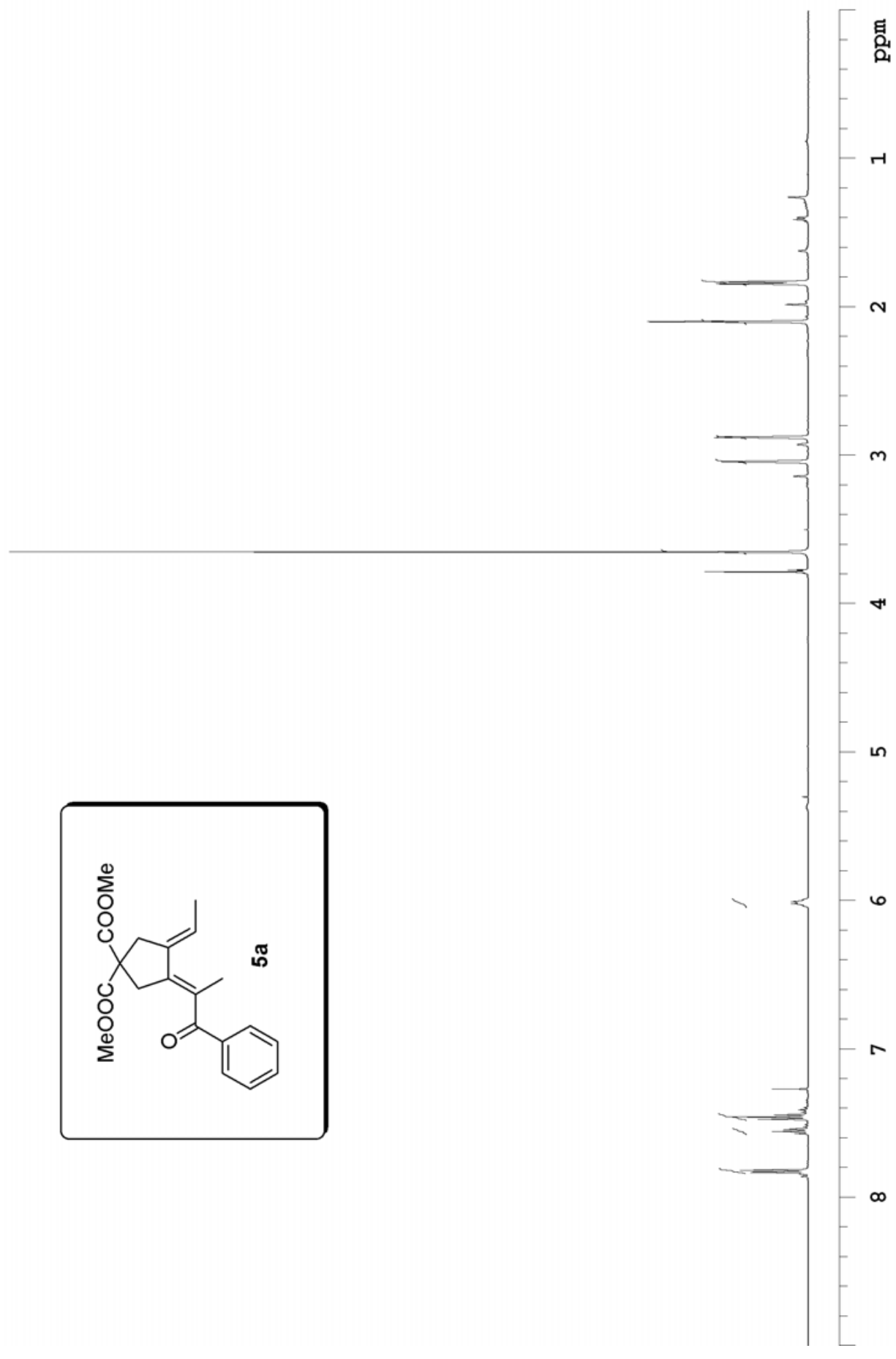




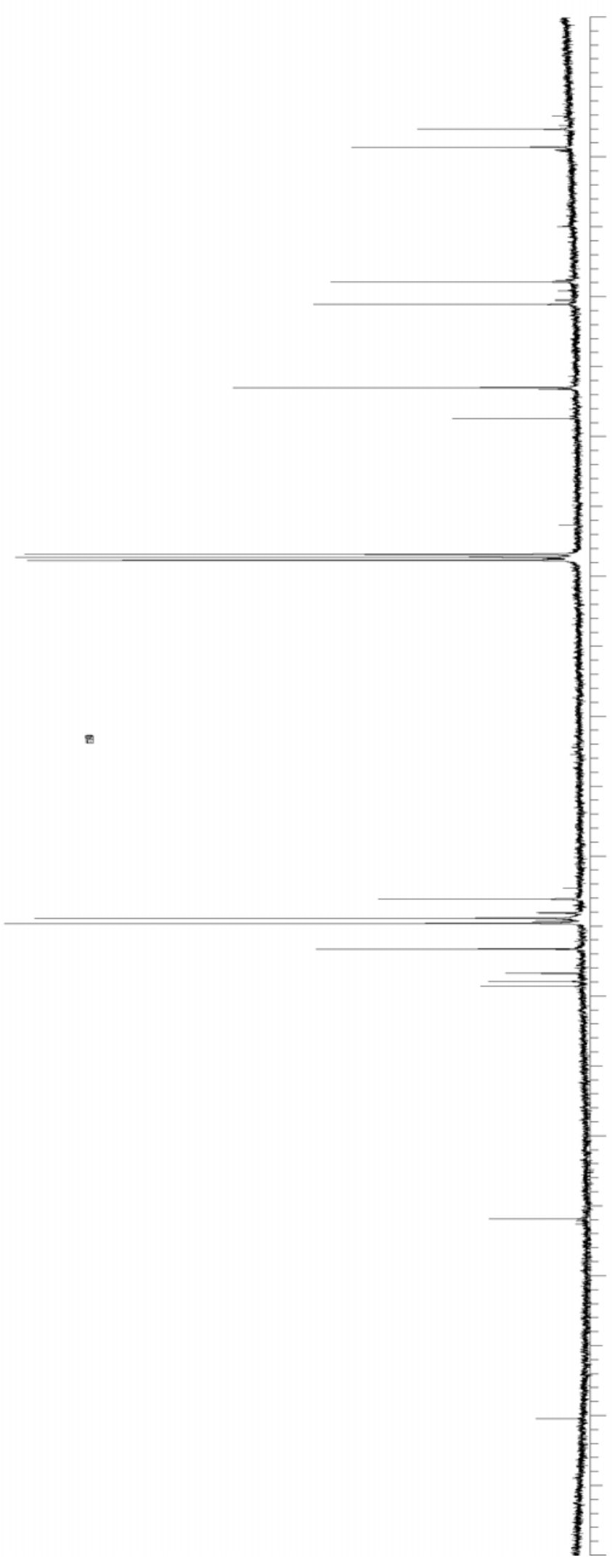




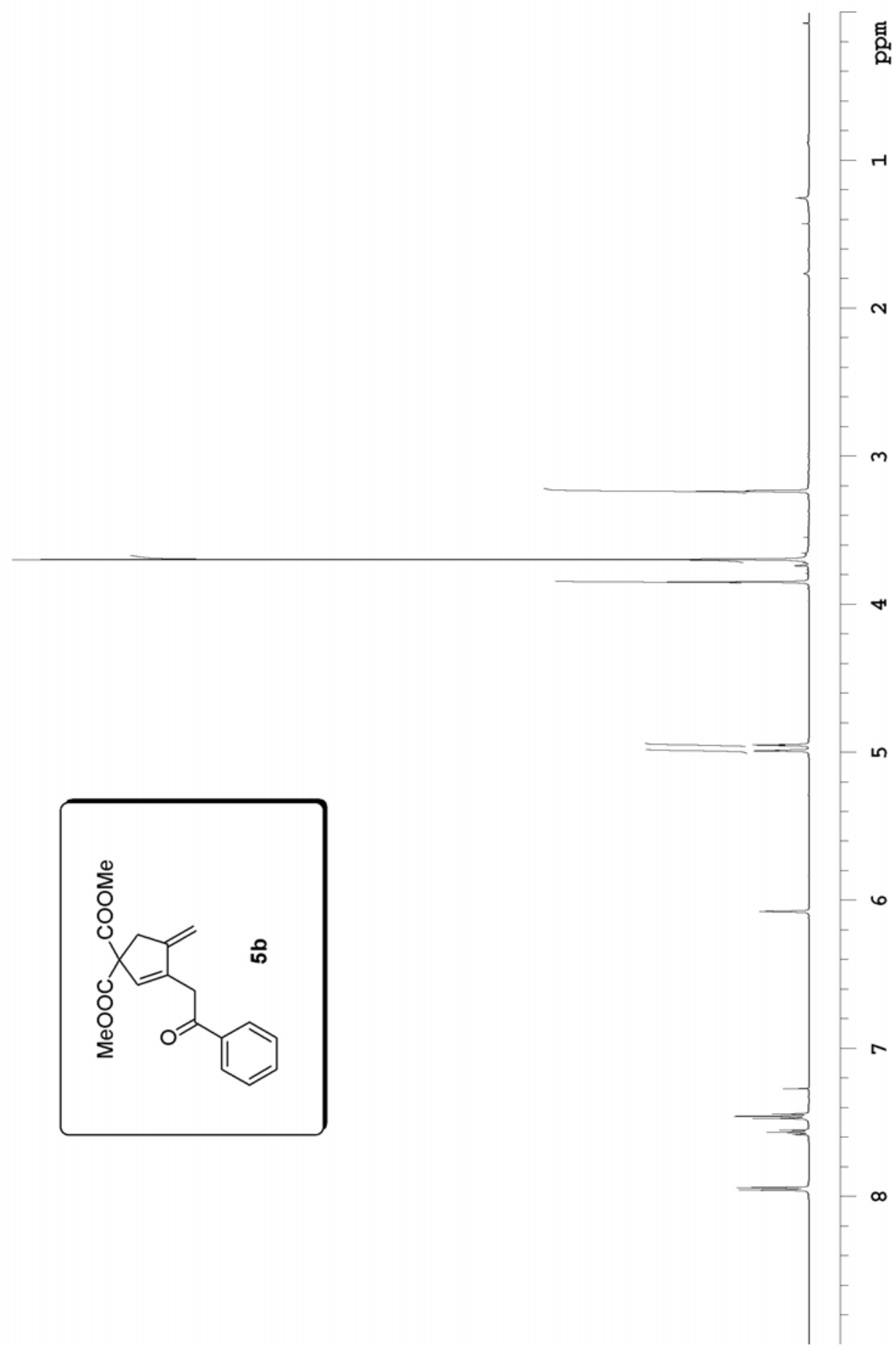




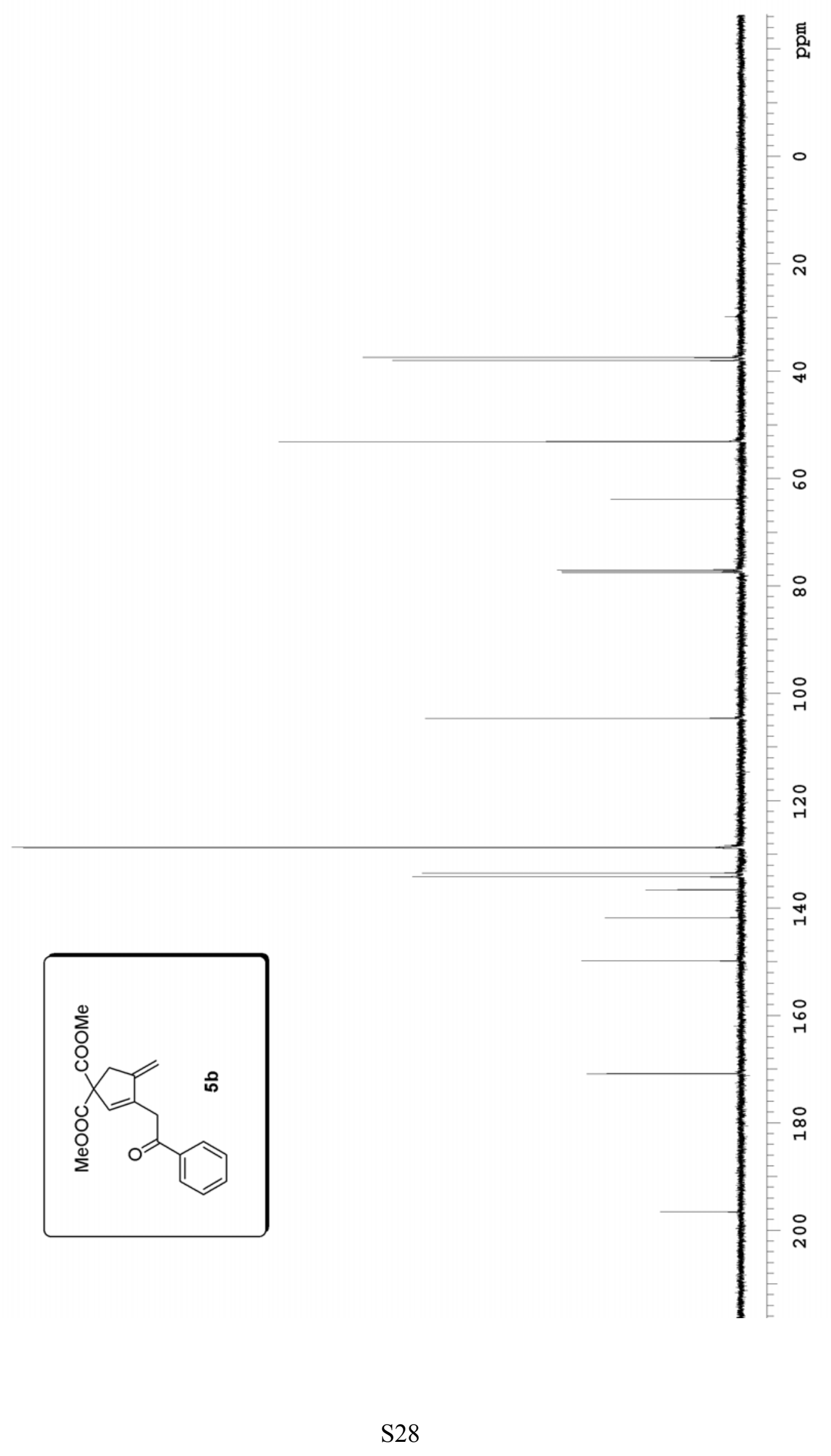




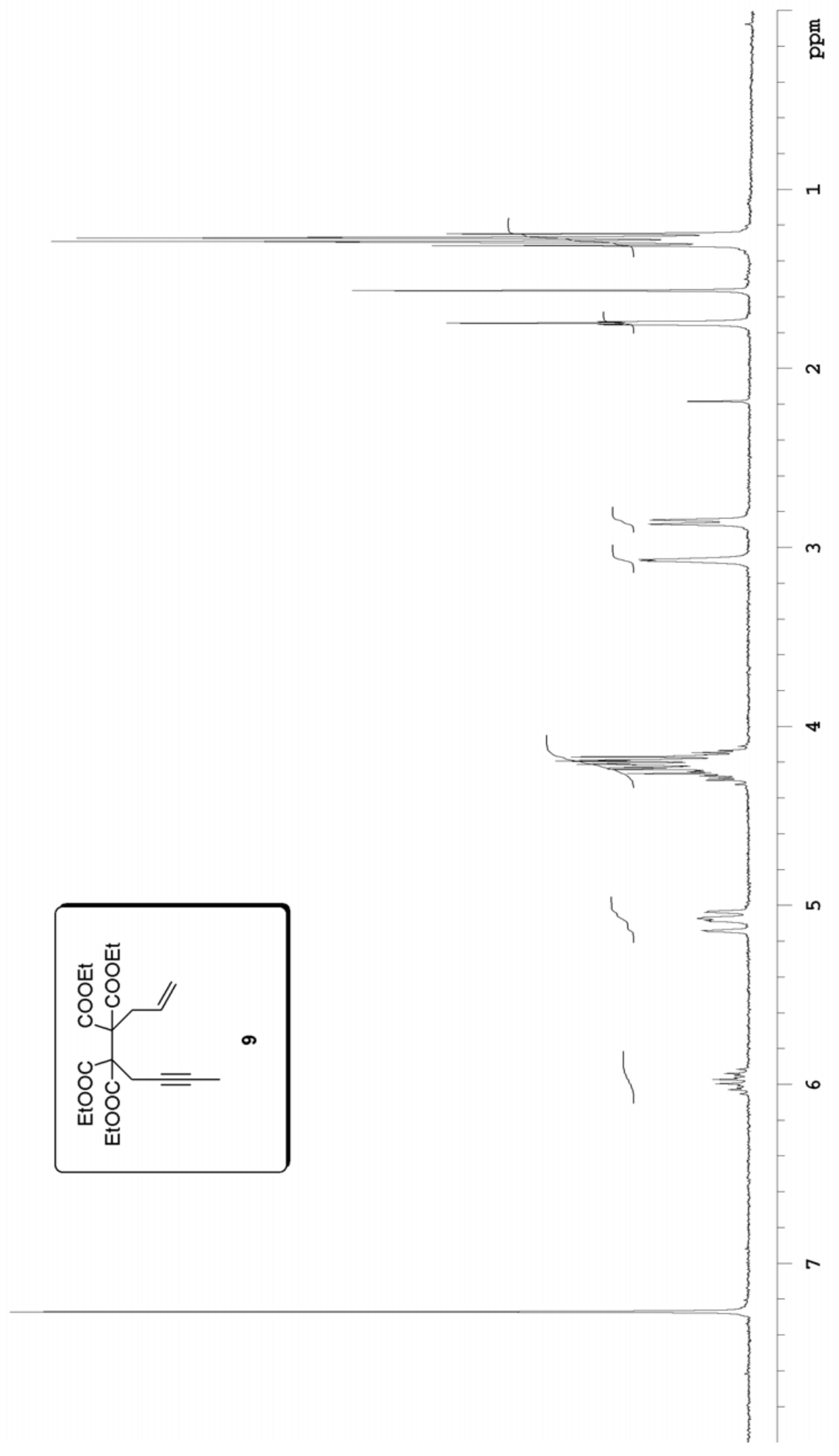




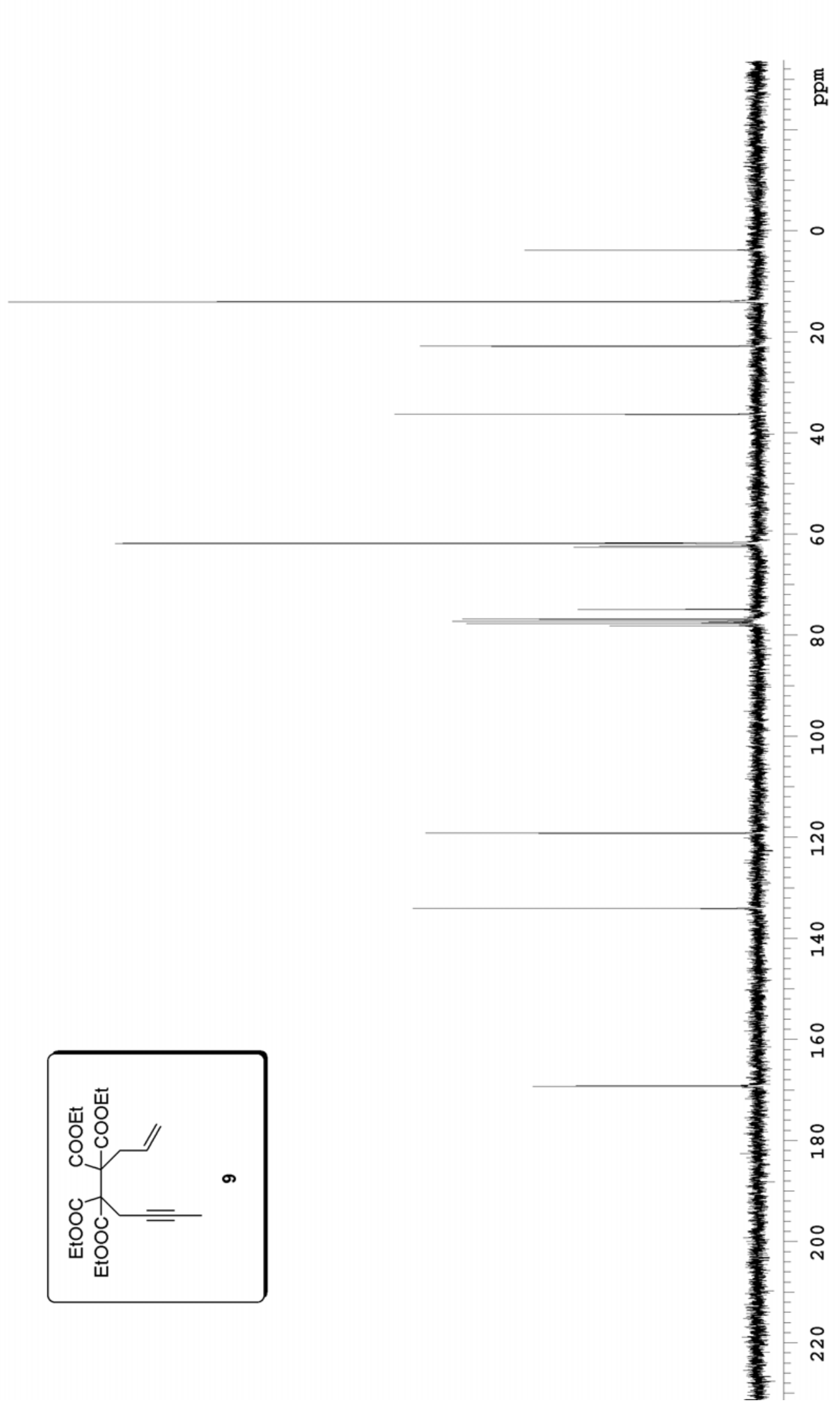




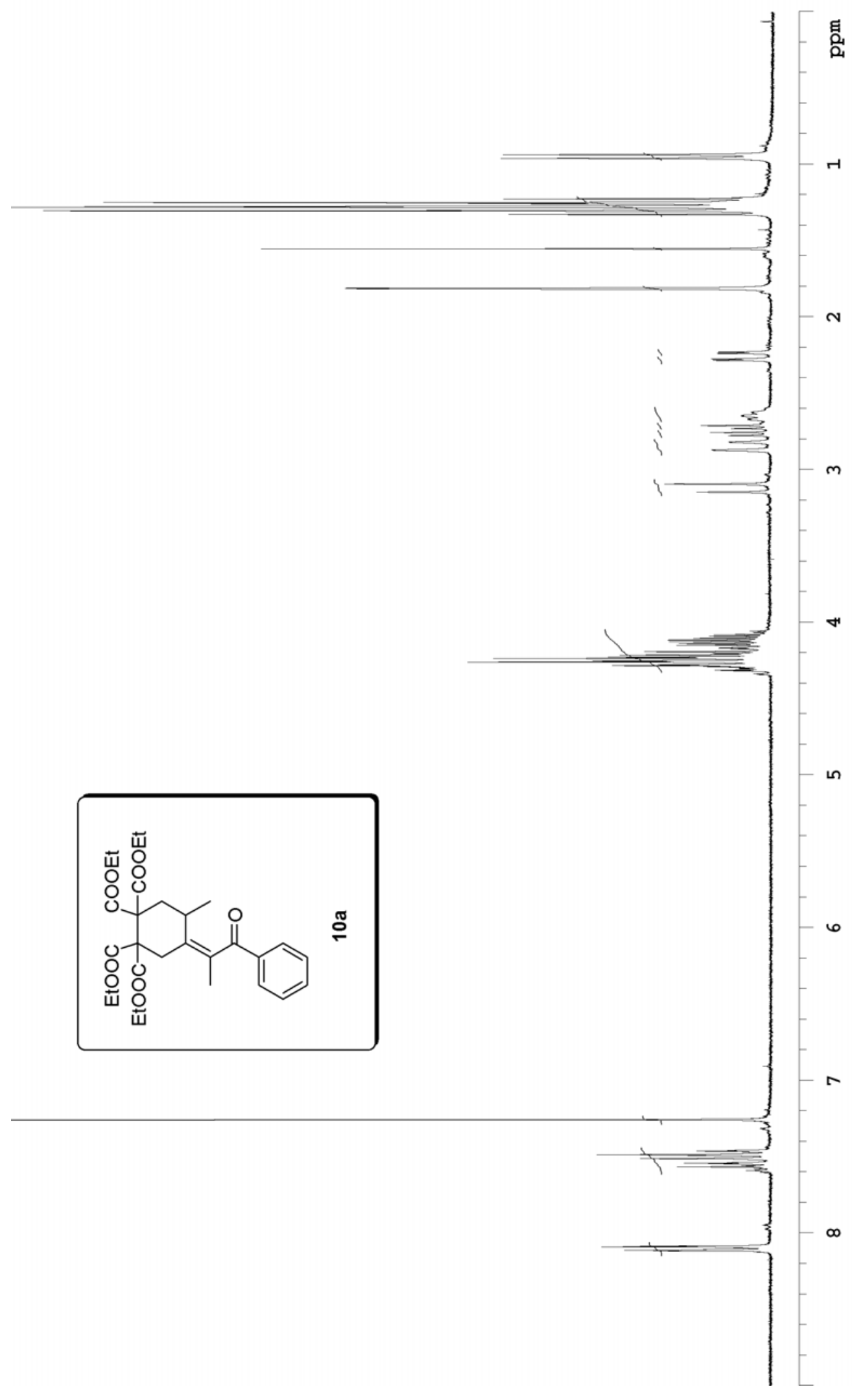




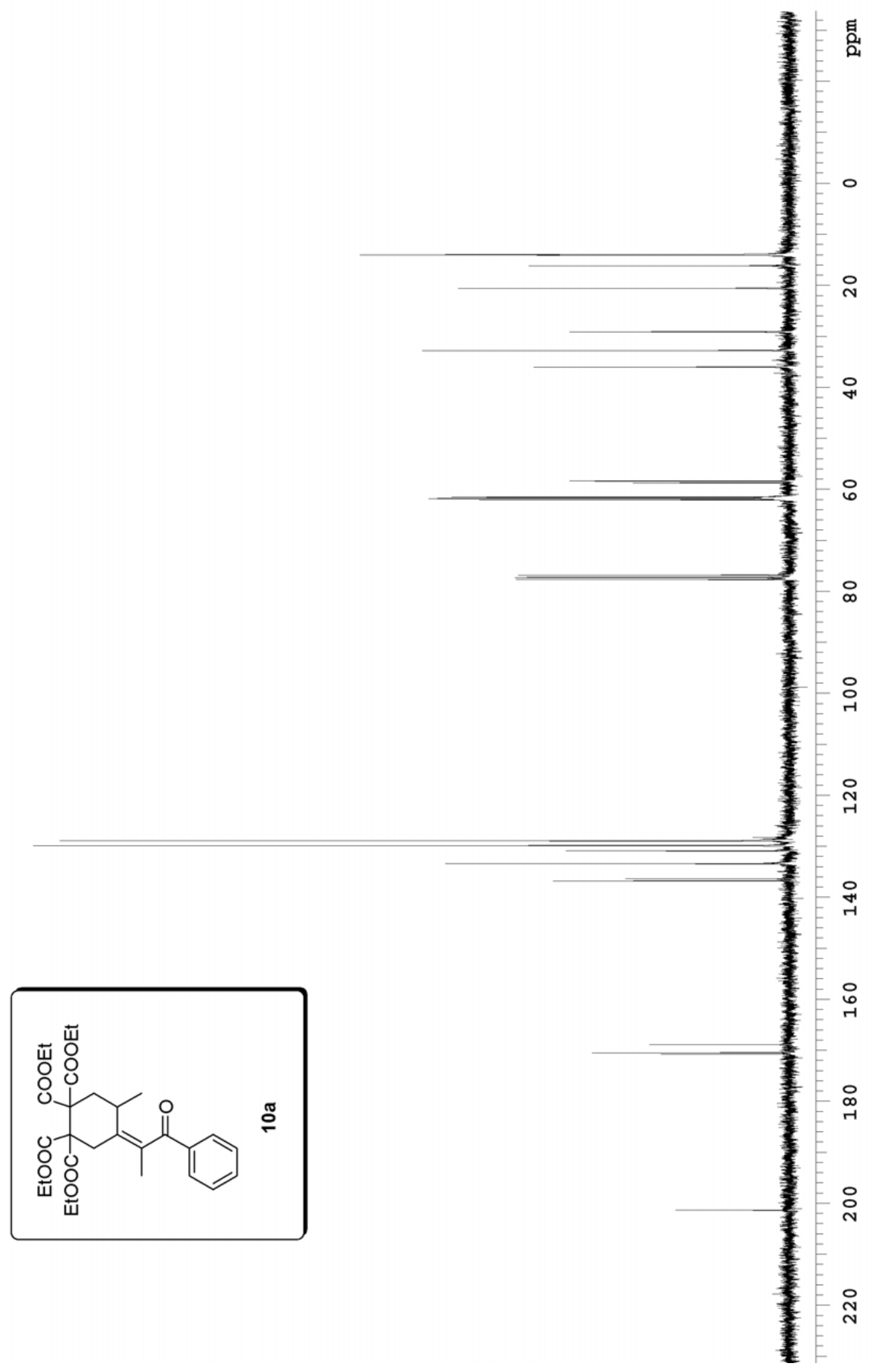

S32 


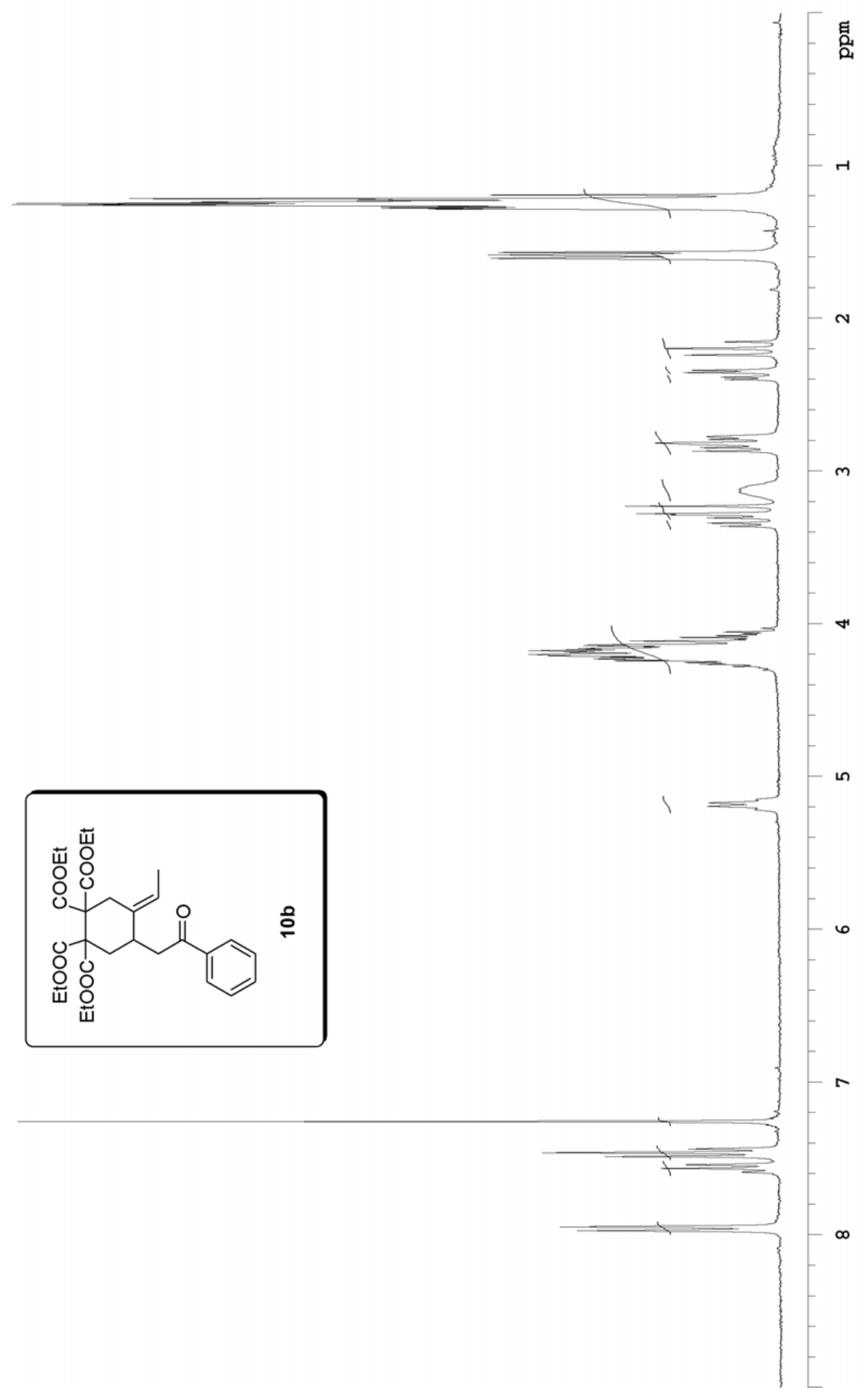




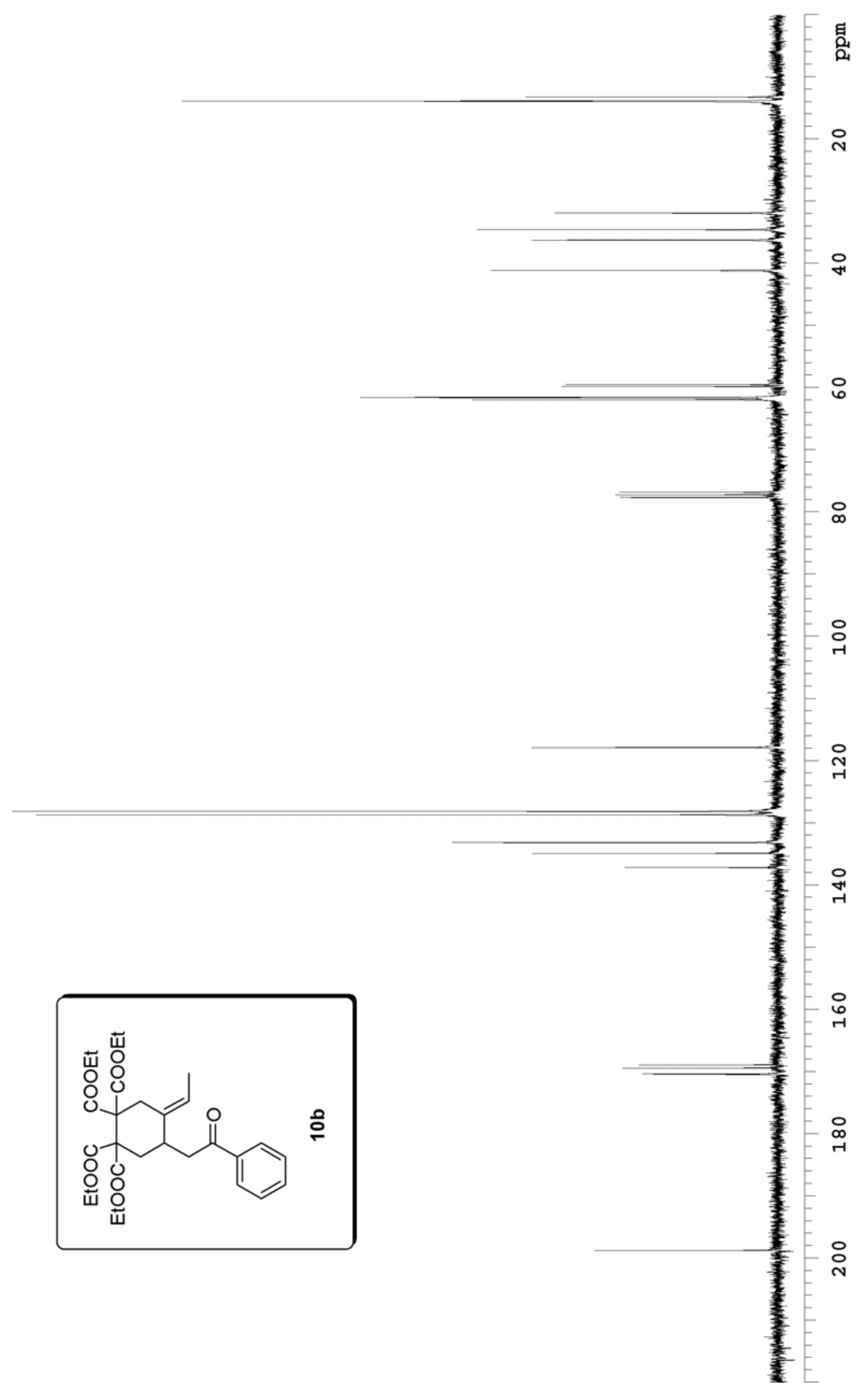




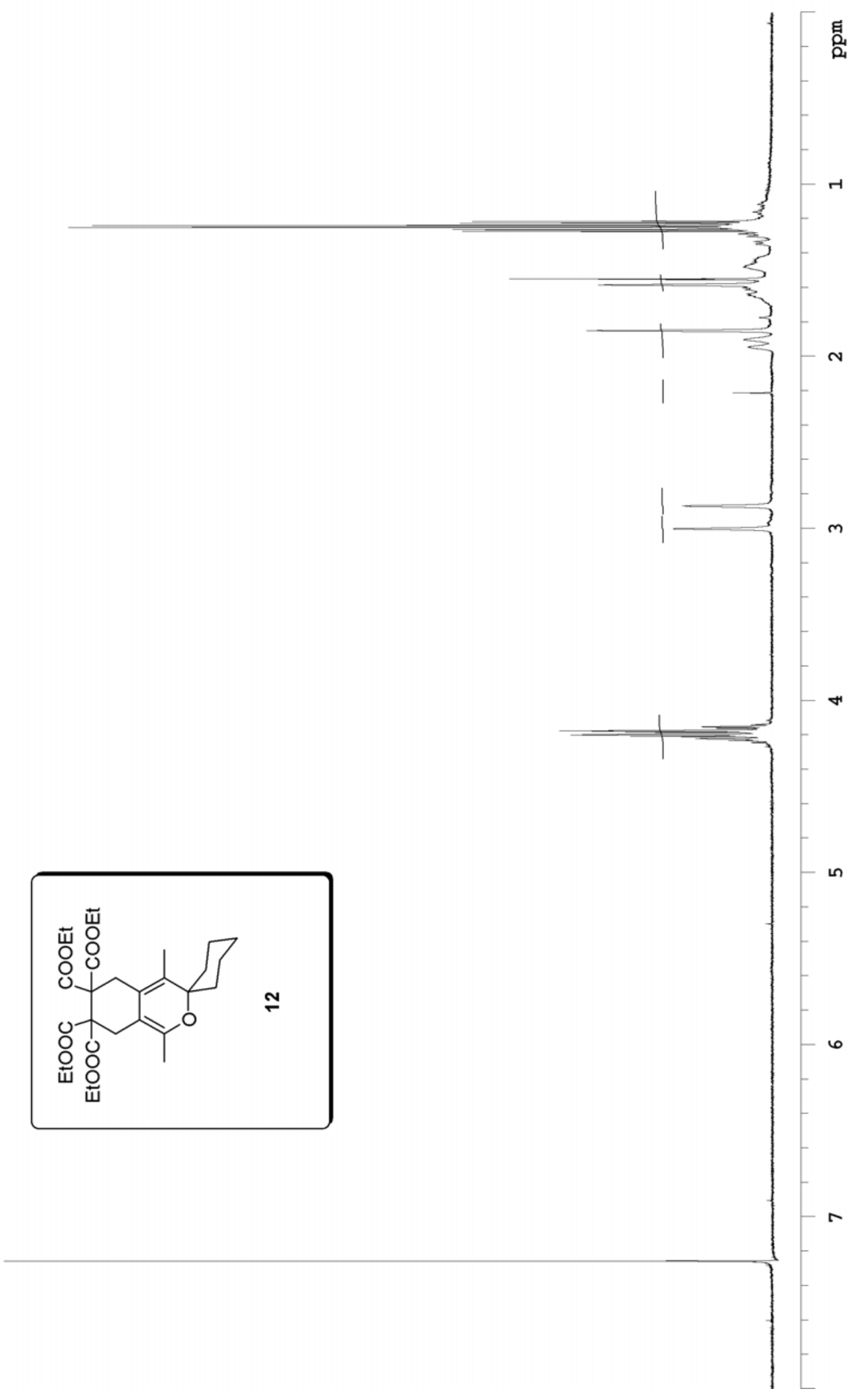




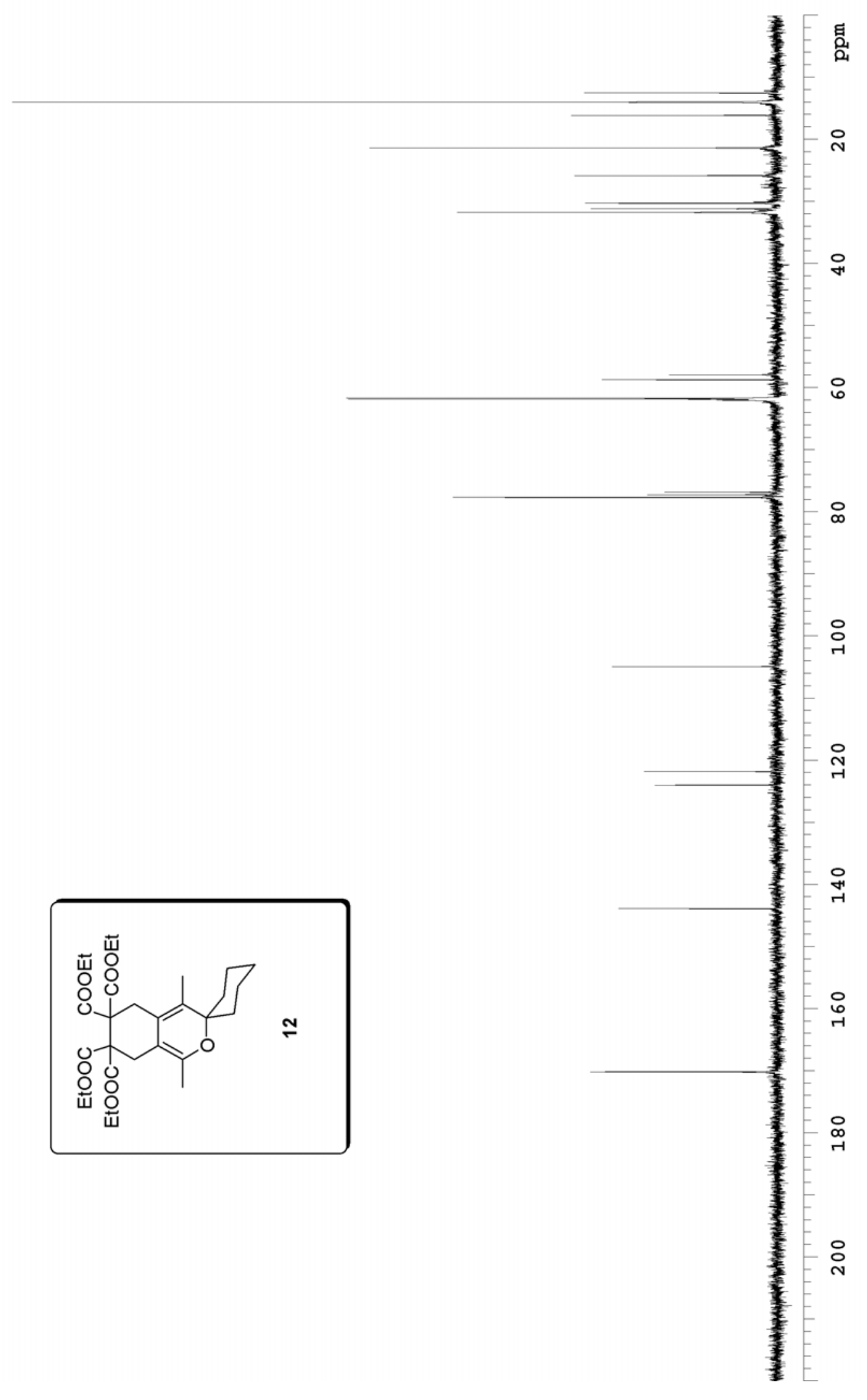

